\title{
The interaction between protostars and their environment: Carbon-bearing species towards low mass protostars
}

\begin{abstract}
J. V. Buckle and G. A. Fuller
Department of Physics, UMIST, PO Box 88, Manchester M60 1QD, UK

Received 9 November 2000 / Accepted 10 September 2001

Abstract. It is clear that outflow activity can have a significant impact on the structure and evolution of material in protostellar environments, but the detailed nature of the interaction between the outflow and surrounding material is not clearly understood. To probe the impact of outflow activity on protostellar core material, in this paper we present observations of $\mathrm{CH}_{3} \mathrm{OH}\left(J_{k}=3_{k} \rightarrow 2_{k}\right)$, c- $\mathrm{C}_{3} \mathrm{H}_{2}\left(J_{k_{-}, k_{+}}=3_{1,2} \rightarrow 2_{2,1}\right)$ and $\mathrm{DCN}(J=2 \rightarrow 1$ multiplet $)$ at $145 \mathrm{GHz}$ towards a sample of Class 0 and Class I sources, to place constraints on the physical and chemical changes which have taken place through the onset of star formation. $\mathrm{CH}_{3} \mathrm{OH}$ was detected towards all of the sources and positions observed. In many sources, the $\mathrm{CH}_{3} \mathrm{OH}$ lines have two velocity components: one broad $(\langle\Delta v\rangle \sim$ $\left.1.5 \mathrm{~km} \mathrm{~s}^{-1}\right)$, and one narrow $\left(\langle\Delta v\rangle \sim 0.7 \mathrm{~km} \mathrm{~s}^{-1}\right)$. Both velocity components of $\mathrm{CH}_{3} \mathrm{OH}$ show significantly $(>10)$ enhanced abundances relative to quiescent dark clouds. These two components are predominantly a feature of the Class 0 sources. These sources also have the broadest lines in both components. $\mathrm{c}_{-} \mathrm{C}_{3} \mathrm{H}_{2}$ was detected towards $87 \%$ of the sources observed and DCN was detected towards $66 \%$ of the sources observed. The narrowest detected component of $\mathrm{c}-\mathrm{C}_{3} \mathrm{H}_{2}$ has similar linewidths to $\mathrm{NH}_{3}$, and is detected towards only a small fraction of positions, implying that only a small number of sources contain undisturbed material. The degree of activity implied by the enhanced abundances in $\mathrm{CH}_{3} \mathrm{OH}$ is larger in the Class 0 sources than the Class I sources. Since the narrower linewidth components also show enhanced abundances in this molecule, we conclude that energetic activity during the star formation process, which may include both outflow and infall processes, affects the apparently quiescent core material very early in the star formation process.
\end{abstract}

Key words. star: formation - ISM: jets and outflows - radio lines: ISM

\section{Introduction}

Most, if not all low mass protostellar sources go through a phase of energetic outflow activity during their evolution (Lada 1982). Outflows are thought to commence very early during protostellar evolution, although their transitory nature, and the lack of detailed observations and theories on the acceleration mechanisms, make the outflow dynamical timescale a poor estimator of protostellar ages (Chernin \& Masson 1995; Parker et al. 1991). The outflows play an important role in theories of star formation, where they contribute to mass loss, angular momentum transport and clearing circumstellar material (Richer et al. 2000; Hayashi et al. 1994; Terebey et al. 1990). In addition, the outflows can drain envelope material into the outflow at large distances from the collimated driving jet (Stahler 1994). The bulk of the outflow material is assumed to consist of swept up ambient material, a view

Send offprint requests to: J. V. Buckle,

e-mail: j.buckle@jach.hawaii.edu supported by evidence from comparisons of outflow and cloud morphology, and from comparisons of outflow and stellar mass (Masson \& Chernin 1993). The outflow can have a significant effect on the structure and evolution of the surrounding core (Parker et al. 1991), with kinetic energies large enough to disrupt and disperse the remnant circumstellar envelope. However, the nature of the interaction between the outflow and surrounding circumstellar material is not clearly understood. As the outflow first breaks out, it sweeps up a significant amount of circumstellar material (Fuller \& Ladd 2002). Thereafter, it is unclear to what extent the outflow continues to interact with the circumstellar material.

The energetic interaction between the outflow and the surrounding circumstellar material has a significant impact on both the chemical composition and physical structure of the protostellar environment (Gibb \& Davis 1998; Lada 1985). Dust grains are processed through shocks, which destroy the grain mantles, and leads to a rich chemical environment surrounding the outflow. This chemical 
Table 1. Molecular line list.

\begin{tabular}{llrr}
\hline Molecule & Transition & $\begin{array}{r}\text { Frequency } \\
\mathrm{MHz}\end{array}$ & $\begin{array}{r}E_{\text {lower }} \\
\mathrm{K}\end{array}$ \\
\hline $\mathrm{CH}_{3} \mathrm{OH}$ & $J_{k}=3_{1} \rightarrow 2_{1} E^{+}$ & 145131.88 & 28.01 \\
$\mathrm{CH}_{3} \mathrm{OH}$ & $J_{k}=3_{2} \rightarrow 2_{2} E^{+}, E^{-}$ & 145126.37 & $28.4,31.9$ \\
$\mathrm{CH}_{3} \mathrm{OH}$ & $J_{k}=3_{0} \rightarrow 2_{0} A^{+}$ & 145103.23 & 6.96 \\
$\mathrm{CH}_{3} \mathrm{OH}$ & $J_{k}=3_{1} \rightarrow 2_{1} E^{-}$ & 145097.47 & 12.34 \\
$\mathrm{CH}_{3} \mathrm{OH}$ & $J_{k}=3_{0} \rightarrow 2_{0} E^{+}$ & 145093.75 & 20.09 \\
$\mathrm{c}-\mathrm{C}_{3} \mathrm{H}_{2}$ & $J_{k_{-}, k_{+}}=3_{1,2} \rightarrow 2,1$ & 145089.60 & 9.09 \\
$\mathrm{DCN}$ & $J=2 \rightarrow 1 F^{\prime}=1 \rightarrow 1$ & 144830.34 & 3.48 \\
$\mathrm{DCN}$ & $J=2 \rightarrow 1 F^{\prime}=3 \rightarrow 2$ & 144828.11 & 3.48 \\
$\mathrm{DCN}$ & $J=2 \rightarrow 1 F^{\prime}=2 \rightarrow 1$ & 144828.00 & 3.48 \\
$\mathrm{DCN}$ & $J=2 \rightarrow 1 F^{\prime}=1 \rightarrow 0$ & 144826.82 & 3.48 \\
$\mathrm{DCN}$ & $J=2 \rightarrow 1 F^{\prime}=2 \rightarrow 2$ & 144826.57 & 3.48 \\
$\mathrm{C}^{18} \mathrm{O}$ & $J=1 \rightarrow 0$ & 109782.17 & 0.00 \\
\hline
\end{tabular}

enrichment can be used as a probe to assess the role of protostellar outflows in clearing circumstellar regions. By observing different molecular transitions which require a range of excitation conditions we can trace different components of the outflow. Methanol is a species which is known to be abundant in the ice mantles on grains. It is volatile, and easily liberated from grain mantles through shock processing (Charnley et al. 1995; Bachiller et al. 1995; Van Dishoeck et al. 1995). The ease with which it is liberated, and the consequent increase in its abundance should make methanol a sensitive probe of the interaction between outflows and quiescent dense gas. In addition, since methanol is present in both quiescent and processed material, the same molecular probe can be used to determine changes in the physical properties due to outflow interactions. The deuterated species DCN, which has hyperfine components in the transitions at radio wavelengths, has been observed in protostellar environments (Van Dishoeck et al. 1995), where it is thought to be released into the gas phase through grain mantle evaporation. This can be used to further constrain the type of chemistry taking place, whether predominantly low temperature gas phase, ice mantle formation or shock processing (Hatchell et al. 1998). The organic ring molecule $\mathrm{c}-\mathrm{C}_{3} \mathrm{H}_{2}$ has been observed in molecular clouds (Lee et al. 1993) and protostellar environments (Bachiller \& Gutiérrez 1997; Tafalla \& Myers 1997), where it has been associated with the central, dense regions surrounding the protostar.

In this paper, we report on observations of $\mathrm{CH}_{3} \mathrm{OH}$, c- $\mathrm{C}_{3} \mathrm{H}_{2}$ and DCN in a sample of 30 protostellar objects. In Sect. 2 we describe the observations and data reduction and in Sect. 3 we describe the results of the observations. In Sect. 4, we describe the different methods used to analyse the data, and report on the results. In Sect. 5, we discuss the interpretation of our results. Finally, we summarise our findings in Sect. 6.

\section{Observations and data reduction}

Single dish observations of $\mathrm{CH}_{3} \mathrm{OH} J_{k}=3_{k} \rightarrow 2_{k}$ transitions at $145 \mathrm{GHz}$ were made using the NRAO (National Radio Astronomy Observatory) $12 \mathrm{~m}$ telescope in February 1999, using the $2 \mathrm{~mm}$ SIS receivers and the MAC (Millimetre Autocorrelator) spectrometer in $4 \mathrm{IF}$ mode with 8000 channels. The spectra were observed using $15 \mathrm{kHz}$ channels, giving a velocity resolution of $\sim 0.03 \mathrm{~km} \mathrm{~s}^{-1}$. The NRAO beam size at this frequency is $43^{\prime \prime}$, and the corrected main beam efficiency of the telescope, $\eta_{\mathrm{m}}^{*}$, is 0.76 . The observations were made in two polarization channels and averaged. The $3_{1,2} \rightarrow 2_{2,1}$ transition of $\mathrm{c}-\mathrm{C}_{3} \mathrm{H}_{2}$ was observed in the same spectrum as the $\mathrm{CH}_{3} \mathrm{OH}$ transitions, while the DCN $2 \rightarrow 1$ hyperfine components were observed in parallel channels, which were offset by $290 \mathrm{MHz}$. Since the $\mathrm{CH}_{3} \mathrm{OH}$ lines could be observed simultaneously, the derived quantities are not subject to relative calibration uncertainties. The weather was good during the observations, giving effective system temperatures of between $170 \mathrm{~K}$ and $200 \mathrm{~K}$. The data were taken in position-switching mode, with offset positions of $\pm 15^{\prime}$. Table 1 lists the molecular lines that we detected, with their associated frequencies and energies.

In addition, observations of $\mathrm{C}^{18} \mathrm{O} J=1 \rightarrow 0$, at $109.78 \mathrm{GHz}$ were taken using the NRAO $12 \mathrm{~m}$ telescope in February 2000. The observations were taken using the $3 \mathrm{~mm}$ receiver in position switching mode, with the MAC in 2IF mode with 16000 channels. These observations were taken in order to constrain the $\mathrm{CH}_{3} \mathrm{OH}$ abundances.

The observations were made at a total of 60 positions toward 18 Class 0 and 12 Class I sources that are known to have associated outflows. Table 2 lists the sources observed with the positions, distances and bolometric temperatures. The sample was chosen to include as many of the very young (Class 0) sources as possible. Several Class I sources, with evidence of molecular outflow emission, were also chosen to be used as a comparison with the Class 0 sources. In addition to observations towards the embedded source, for those sources which showed strong methanol lines, we observed at several positions around the source, where previous observations have shown peaks in the CO outflow emission. These positions are listed in Table 2 as arcsecond offsets from the source position above them. We refer to the positions centred on CO outflow peaks as "outflow" positions, and the positions centred on the protostellar source as "core" positions.

Figures 1 and 2 show sample spectra from the protostellar sources. In Fig. 1, spectra were chosen to show the range of linewidths and intensities detected for $\mathrm{CH}_{3} \mathrm{OH}$ and $\mathrm{c}_{-} \mathrm{C}_{3} \mathrm{H}_{2}$ molecules. The velocity scales are set to the velocity of the $J_{k}=3_{0} \rightarrow 2_{0} A^{+}$transition. Figure 2 shows examples of the DCN detections.

Data reduction was carried out using the radio data reduction software CLASS. Despite the variety of line profiles detected, we find that Gaussian fitting to the line profile provides a good fit to the data, and allows a simple comparison of linewidths and integrated intensities. 


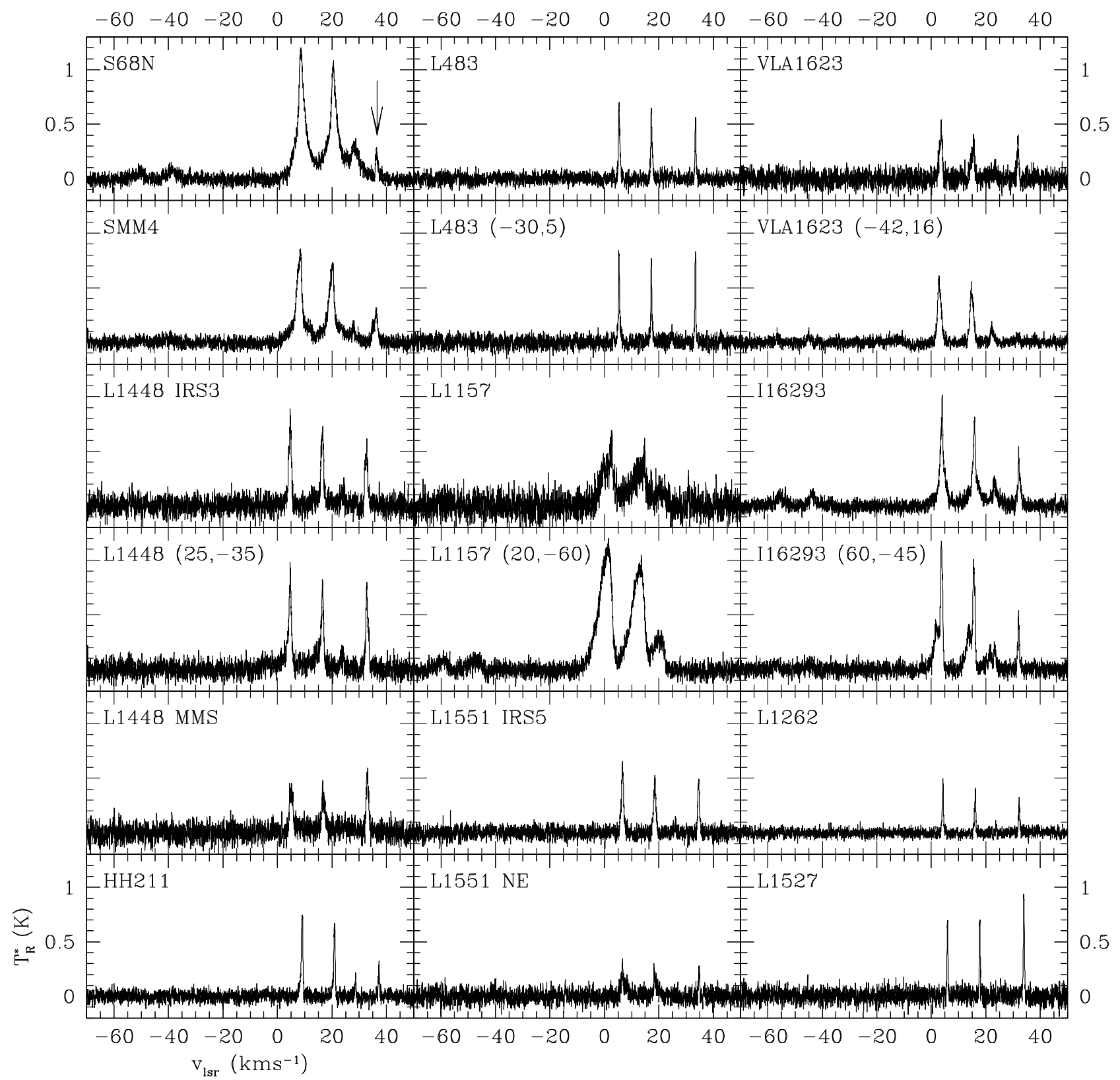

Fig. 1. Spectra from a sample of sources, showing the spectra obtained at $145 \mathrm{GHz}\left(\mathrm{CH}_{3} \mathrm{OH}, \mathrm{c}-\mathrm{C}_{3} \mathrm{H}_{2}\right)$. An arrow marks the position of the $\mathrm{c}_{-} \mathrm{C}_{3} \mathrm{H}_{2}$ transition in the spectrum of $\mathrm{S} 68 \mathrm{~N}$. All spectra are plotted to the same velocity and temperature scales.

For c- $\mathrm{C}_{3} \mathrm{H}_{2}$, single or two component Gaussians were fitted to the data using the CLASS routines. The choice of single or double component Gaussians was made on the basis of producing the best fit to the data. If a double component line that produced the best fit was the sum of two very similar velocity and linewidth components, then a single component fit was made. For DCN, CLASS routines that account for the multiplet transitions were used. For $\mathrm{CH}_{3} \mathrm{OH}$, we used a separate fitting procedure based on single or double Gaussian components. Since the separation of the $\mathrm{K}$ transitions is fixed by the structure of the molecule, each of the Gaussian components had 7 free parameters: its width, its velocity and the intensity in each of the $5 \mathrm{~K}$ transitions. Using this procedure, we hope to obtain a more useful fit to the data than by freely fitting all the lines in the spectra.

\section{Detections}

\section{1. $\mathrm{CH}_{3} \mathrm{OH}$}

Methanol was detected towards all of the observed positions. The spectra shown in Fig. 1 demonstrate the range of linewidths, line profiles and peak temperatures detected towards the protostellar sources. Towards some core positions, such as L1527 and L483, the lines are narrow, whereas cores such as S68N and SMM4 have large blue and red wings. In some outflow positions, such 
Table 2. Summary of sources observed. Bolometric temperatures, $T_{\text {bol }}$, and source distances, $d$, are obtained from the current literature.

\begin{tabular}{|c|c|c|c|c|c|c|c|c|c|}
\hline Source & $\begin{array}{r}T_{\text {bol }} \\
(\mathrm{K})\end{array}$ & $\begin{array}{r}d \\
(\mathrm{pc})\end{array}$ & $\begin{array}{r}\mathrm{RA} \\
1950\end{array}$ & $\begin{array}{r}\text { Dec } \\
1950\end{array}$ & Source & $\begin{array}{l}T_{\text {bol }} \\
(\mathrm{K})\end{array}$ & $\begin{array}{r}d \\
(\mathrm{pc})\end{array}$ & $\begin{array}{r}\mathrm{RA} \\
1950\end{array}$ & $\begin{array}{r}\text { Dec } \\
1950\end{array}$ \\
\hline L1448 IRS3 & 70 & 300 & $03: 22: 31.9$ & $30: 34: 48.0$ & continuum peak & & & $45^{\prime \prime}$ & $6^{\prime \prime}$ \\
\hline L1448 NW & & & $-17^{\prime \prime}$ & $16^{\prime \prime}$ & red lobe peak & & & $-71^{\prime \prime}$ & $36^{\prime \prime}$ \\
\hline blue lobe peak & & & $25^{\prime \prime}$ & $-35^{\prime \prime}$ & LFAM1 & 174 & 160 & $16: 23: 20.3$ & $-24: 16: 06.6$ \\
\hline red lobe peak & & & $57^{\prime \prime}$ & $-110^{\prime \prime}$ & I16244-2432 & 157 & 160 & $16: 24: 26.1$ & $-24: 32: 51.0$ \\
\hline $\mathrm{L} 1448 \mathrm{mms}$ & 56 & 300 & $03: 22: 34.3$ & $30: 33: 35.0$ & blue lobe peak & & & $35^{\prime \prime}$ & $15^{\prime \prime}$ \\
\hline red lobe peak & & & $33^{\prime \prime}$ & $-77^{\prime \prime}$ & I16293-2422 & 43 & 160 & $16: 29: 20.9$ & $-24: 22: 13.0$ \\
\hline I03282+3035 & 26 & 300 & $03: 28: 15.2$ & $30: 35: 14.0$ & red lobe peak & & & $60^{\prime \prime}$ & $-45^{\prime \prime}$ \\
\hline HH211 & 30 & 300 & 03:40:48.7 & $31: 51: 24.0$ & L483 & 48 & 200 & $18: 14: 50.6$ & $-04: 40: 49.0$ \\
\hline red lobe peak & & & $-14^{\prime \prime}$ & $7^{\prime \prime}$ & blue lobe peak & & & $-30^{\prime \prime}$ & \\
\hline blue lobe peak & & & $18^{\prime \prime}$ & $-9^{\prime \prime}$ & red lobe peak & & & $40^{\prime \prime}$ & $-5^{\prime \prime}$ \\
\hline quiescent cloud & & & $41^{\prime \prime}$ & $14^{\prime \prime}$ & L483 SW & & & $-30^{\prime \prime}$ & $-60^{\prime \prime}$ \\
\hline blue lobe peak & & & $41^{\prime \prime}$ & $-14^{\prime \prime}$ & S68N & 40 & 300 & $18: 27: 15.6$ & 01:14:42.0 \\
\hline IRAM04191+1522 & 18 & 140 & 04:19:06.4 & $15: 22: 45.0$ & quiescent cloud & & & $46^{\prime \prime}$ & $66^{\prime \prime}$ \\
\hline red lobe peak & & & $6^{\prime \prime}$ & $17^{\prime \prime}$ & SMM1 & 51 & 300 & $18: 27: 17.5$ & 01:12:13.3 \\
\hline Haro6-10 & 242 & 140 & $04: 26: 21.9$ & $24: 26: 29.0$ & SMM4 & 43 & 300 & $18: 27: 24.8$ & 01:11:08.0 \\
\hline L1551IRS5 & 97 & 140 & $04: 28: 40.2$ & 18:01:42.0 & B335 & 37 & 250 & $19: 34: 35.7$ & $07: 27: 20.0$ \\
\hline L1551NE & 75 & 140 & $04: 28: 50.8$ & $18: 02: 11.0$ & blue lobe peak & & & $-60^{\prime \prime}$ & $24^{\prime \prime}$ \\
\hline blue lobe peak & & & $-168^{\prime \prime}$ & $-96^{\prime \prime}$ & L1152 & 72 & 440 & $20: 35: 19.4$ & $67: 42: 30.0$ \\
\hline L1535 & 152 & 140 & $04: 32: 33.4$ & $24: 02: 13.0$ & blue lobe peak & & & $15^{\prime \prime}$ & $30^{\prime \prime}$ \\
\hline TMR-1 & 144 & 140 & 04:36:09.7 & $25: 47: 29.0$ & L1157 & 62 & 440 & $20: 38: 39.6$ & $67: 51: 33.0$ \\
\hline L1527 & 59 & 140 & $04: 36: 49.3$ & $25: 57: 16.0$ & blue lobe peak & & & $20^{\prime \prime}$ & $-60^{\prime \prime}$ \\
\hline RNO43 & 33 & 400 & 05:29:30.6 & $12: 47: 25.0$ & $\mathrm{I} 20126+4104$ & (0) & 1700 & 20:12:41.0 & 41:04:21.0 \\
\hline HH25 & 34 & 400 & $05: 43: 33.7$ & $-00: 16: 05.0$ & L1174 & (I) & 440 & $20: 59: 42.1$ & 68:00:12.0 \\
\hline red lobe peak & & & $224^{\prime \prime}$ & $30^{\prime \prime}$ & L1251B & 91 & 200 & $22: 37: 40.8$ & $74: 55: 50.0$ \\
\hline HH111 & 38 & 460 & 05:49:09.3 & $02: 47: 48.0$ & blue lobe peak & & & $-81^{\prime \prime}$ & $85^{\prime \prime}$ \\
\hline VLA1623 & 30 & 160 & $16: 23: 24.9$ & $-24: 17: 46.0$ & red lobe peak & & & $128^{\prime \prime}$ & $-50^{\prime \prime}$ \\
\hline red lobe peak & & & $-42^{\prime \prime}$ & $16^{\prime \prime}$ & CepE & $(0)$ & 730 & 23:01:10.1 & $61: 26: 16.0$ \\
\hline blue lobe peak & & & $45^{\prime \prime}$ & $-24^{\prime \prime}$ & blue lobe peak & & & $-24^{\prime \prime}$ & $-22^{\prime \prime}$ \\
\hline continuum peak & & & $16^{\prime \prime}$ & $76^{\prime \prime}$ & red lobe peak & & & $4^{\prime \prime}$ & $17^{\prime \prime}$ \\
\hline continuum peak & & & $16^{\prime \prime}$ & $31^{\prime \prime}$ & L1262 & 104 & 200 & $23: 23: 48.7$ & 74:01:08.0 \\
\hline
\end{tabular}

as $\operatorname{L} 1448\left(25^{\prime \prime},-35^{\prime \prime}\right)$ and $\operatorname{L1} 157\left(20^{\prime \prime},-60^{\prime \prime}\right)$, asymmetric wings are observed. Other outflow positions, such as VLA1623 $\left(-42^{\prime \prime}, 16^{\prime \prime}\right)$, do not have any obvious asymmetric broadening.

The $J_{k}=3_{0} \rightarrow 2_{0} A^{+}\left(E_{l} \sim 7 \mathrm{~K}\right)$ and $J_{k}=3_{1} \rightarrow 2_{1} E^{-}$ $\left(E_{l} \sim 12 \mathrm{~K}\right)$ transitions, which lie between 0 and $25 \mathrm{kms}^{-1}$ in the spectra, were detected towards all of the observed positions, and are the strongest $\mathrm{CH}_{3} \mathrm{OH}$ lines in all of the sources. The $J_{k}=3_{1} \rightarrow 2_{1} E^{+}\left(E_{l} \sim 28 \mathrm{~K}\right)$ and $J_{k}=3_{2} \rightarrow 2_{2} E^{+/-}\left(E_{l} \sim 30 \mathrm{~K}\right)$ transitions, which lie between -60 and $-30 \mathrm{kms}^{-1}$, were much weaker, and were detected towards only $\sim 12 \%$ of positions. The $\mathrm{CH}_{3} \mathrm{OH}$ $J_{k}=3_{0} \rightarrow 2_{0} E^{+}\left(E_{l} \sim 20 \mathrm{~K}\right)$ transition is also weaker than the lower lying transitions, and was detected towards $\sim 65 \%$ of positions. This line is brighter in the sources which also have emission from the higher lying $\left(E_{l}>\right.$ $20 \mathrm{~K}$ ) transitions. The few sources that show unambiguous emission in all of the transitions are all Class 0 sources. In IRAS 16293-2422, all five transitions are detected towards both core and outflow positions. In S68N, all five transitions are detected towards the core positions (we did not observe an outflow position for this source). For two other sources, L1157 and VLA1623, the outflow positions show 5 transitions, whereas the core positions show only the three lower lying transitions, evidence of warmer material in the outflow.

\section{2. $c-\mathrm{C}_{3} \mathrm{H}_{2}$}

c- $\mathrm{C}_{3} \mathrm{H}_{2}$ was detected towards $\sim 87 \%$ of the positions observed. This molecule has narrow linewidths towards the majority of sources. However, there are exceptions, such as SMM4, where there is an obvious broad component to the line. Emission from $\mathrm{c}-\mathrm{C}_{3} \mathrm{H}_{2}$ tends to have larger peak intensities towards those positions that show narrow $\mathrm{CH}_{3} \mathrm{OH}$ linewidths, such as $\mathrm{L} 1527$ and L483, and is weaker, if detected, towards positions where $\mathrm{CH}_{3} \mathrm{OH}$ lines have broad components, such as S68N and L1157 (Fig. 1). The positions where we do not detect $\mathrm{c}-\mathrm{C}_{3} \mathrm{H}_{2}$ emission are all outflow positions, with the exception of IRAS 162442432 , where we do not detect $\mathrm{c}-\mathrm{C}_{3} \mathrm{H}_{2}$ emission towards either core or outflow positions.

\section{3. $D C N$}

The DCN hyperfine component lines are weaker than the $\mathrm{CH}_{3} \mathrm{OH}$ or $\mathrm{c}-\mathrm{C}_{3} \mathrm{H}_{2}$ lines in our sources, as shown in Fig. 2. Emission from DCN is detected towards both core and 


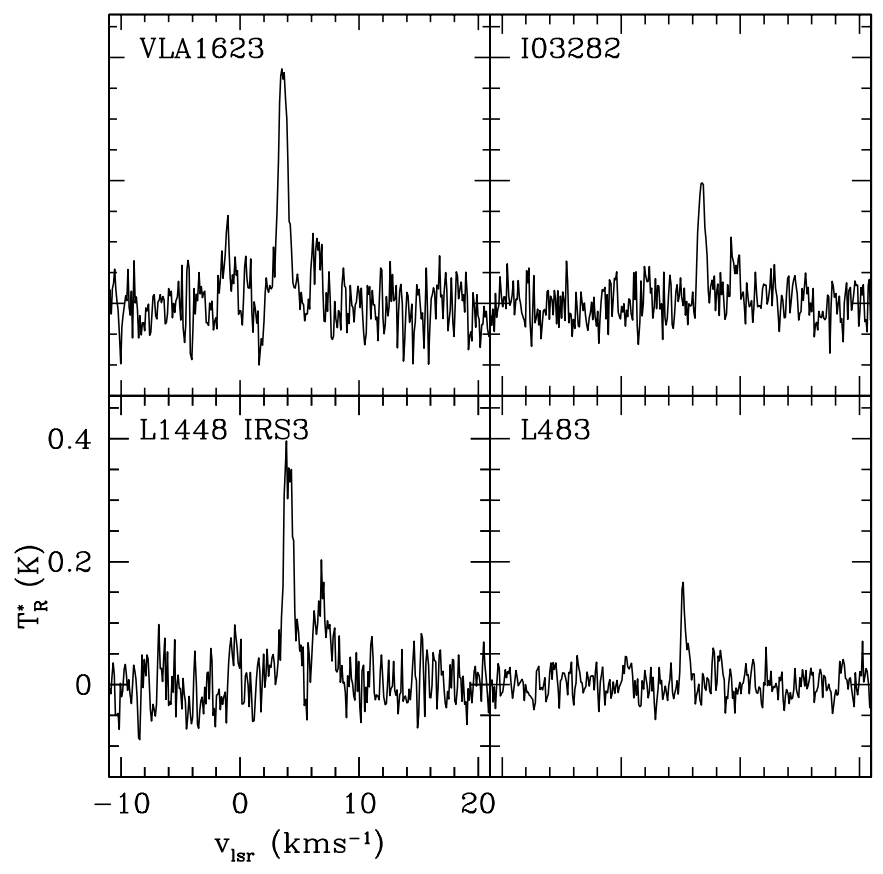

Fig. 2. Spectra from a sample of sources, showing the spectra obtained at $144.8 \mathrm{GHz}(\mathrm{DCN})$. The spectra are plotted to the same velocity and temperature scales, and have been smoothed in order to better display the hyperfine components.

outflow positions, in $\sim 66 \%$ of the positions observed. In those sources where emission from $\mathrm{c}-\mathrm{C}_{3} \mathrm{H}_{2}$ and DCN is not detected, the methanol lines are also weaker. However, towards $\mathrm{L} 1527$, the $\mathrm{CH}_{3} \mathrm{OH}$ and $\mathrm{c}_{-} \mathrm{C}_{3} \mathrm{H}_{2}$ lines are bright, but we did not detect emission from DCN.

\section{Analysis}

\subsection{Linewidths and velocities}

Linewidths, velocities, intensities and associated uncertainties are shown in Table 3. The integrated intensity is calculated from the Gaussian fits to the data, using:

$\int T_{\mathrm{R}}^{*} \mathrm{~d} v=1.06 T_{\mathrm{R}}^{*} \Delta v$

and the corresponding uncertainties are calculated from uncertainties in the linewidths $(\Delta v)$ and peak amplitudes $\left(T_{\mathrm{R}}^{*}\right)$.

\subsubsection{Number of detected line components}

For the sources which have two detectable components, the Gaussian fits generally result in one component which is broader than the other, although a few sources have two components which are distinguishable by a difference in velocity rather than linewidth. For convenience, we label linewidths as single if fitted with one Gaussian, and as broad and narrow if fitted with two Gaussians. For a few sources (e.g. L1448NW), the difference in broad and narrow linewidths may be as little as $0.1 \mathrm{~km} \mathrm{~s}^{-1}$.
The detection of two components in the lines is not a function of distance to the source, as is demonstrated in Figs. 3a-c. The sources at the largest distances are as likely to have two detectable components as the nearer sources. Additionally, we do not see a significant difference in the widths of the lines that we detect as a function of distance to the source (Figs. $3 \mathrm{~d}-\mathrm{e}$ ).

Over half of the positions observed (57\%) have two detectable components in the $\mathrm{CH}_{3} \mathrm{OH}$ emission. Class 0 cores and outflows have the highest fraction of two component lines: $70 \%$ of the outflows and $62 \%$ of the cores for Class 0 compared to $33 \%$ and $20 \%$ for Class I core and outflow positions respectively. Only $\sim 32 \%$ of the sources that have detectable $\mathrm{c}_{-} \mathrm{C}_{3} \mathrm{H}_{2}$ emission show two components in the lines from this molecule, irrespective of class or position.

\subsubsection{Linewidths}

Figure 4 displays the distribution of the broad, narrow and single component linewidths detected towards the sources. Also shown on the plots is the weighted average of the linewidths, over all observed positions, for each component. The weighted average of the linewidths by core and outflow position are listed in Table 4 for $\mathrm{CH}_{3} \mathrm{OH}, \mathrm{c}-\mathrm{C}_{3} \mathrm{H}_{2}$ and DCN. The broadest lines are the broad component $\mathrm{CH}_{3} \mathrm{OH}$ transitions (Fig. 4a), with an average linewidth of $1.5 \mathrm{~km} \mathrm{~s}^{-1}$. The narrowest lines are the narrow component c- $\mathrm{C}_{3} \mathrm{H}_{2}$ transitions (Fig. 4f), with an average linewidth of $0.4 \mathrm{~km} \mathrm{~s}^{-1}$. All of the other components of the molecular transitions have similar average linewidths, of between $0.5-0.7 \mathrm{~km} \mathrm{~s}^{-1}$ (Figs. 4b-e, Table 4).

Perhaps surprisingly, we detect the broadest $\mathrm{CH}_{3} \mathrm{OH}$ lines towards core positions, rather than outflow positions. This is also true for the $\mathrm{c}-\mathrm{C}_{3} \mathrm{H}_{2}$ single component lines. On the other hand, outflow positions have broader $\mathrm{c}-\mathrm{C}_{3} \mathrm{H}_{2}$ broad components, and DCN single components.

When the linewidths for individual sources are compared, as in Fig. 5, we see that positions with broader $\mathrm{CH}_{3} \mathrm{OH}$ linewidths are also likely to have broader $\mathrm{c}-\mathrm{C}_{3} \mathrm{H}_{2}$ and DCN linewidths. As stated previously, the detection of broad lines does not appear to be correlated with the distance to the source (Fig. 3).

\subsubsection{Central velocities}

The sources with two components in $\mathrm{CH}_{3} \mathrm{OH}$ and $\mathrm{c}-\mathrm{C}_{3} \mathrm{H}_{2}$ have central velocities for the broad and narrow component that are similar for each molecule. In most cases, the values lie within a few tenths of a $\mathrm{km} \mathrm{s}^{-1}$, as is illustrated in Fig. 6. The narrow $\mathrm{CH}_{3} \mathrm{OH}$ components (Fig. 6a) are both red and blue-shifted with respect to corresponding broad $\mathrm{CH}_{3} \mathrm{OH}$ components, with differences $<1 \mathrm{~km} \mathrm{~s}^{-1}$. There are a few sources which show larger differences in central velocities between the broad and narrow components, including IRAS $16293(60,-45)$ and L1157. In these 
Table 3. Velocities and intensities from Gaussian fits to the data. Values of $\int T_{\mathrm{R}}^{*} \mathrm{~d} v$ are given for the $J_{k}=3_{0} \rightarrow 2_{0} A^{+}$transition for $\mathrm{CH}_{3} \mathrm{OH}$, which is the strongest line in this spectrum. For DCN, values are quoted for the main hyperfine component, which must be multiplied by 2.146 to get the total intensity in the transition. Uncertainties are quoted in parentheses. Offsets in the source names are in arcsec.

\begin{tabular}{|c|c|c|c|c|c|c|c|}
\hline Source & Molecule & $\begin{array}{r}\Delta v \\
\mathrm{~km} \mathrm{~s}^{-1}\end{array}$ & $\begin{array}{r}v \text { (Broad/Single) } \\
\mathrm{km} \mathrm{s}^{-1}\end{array}$ & $\begin{array}{r}\int T_{\mathrm{R}}^{*} \mathrm{~d} v \\
\mathrm{~K} \mathrm{~km} \mathrm{~s}^{-1}\end{array}$ & $\begin{array}{r}\Delta v \\
\mathrm{~km} \mathrm{~s}^{-1}\end{array}$ & $\begin{array}{r}v \text { (Narrow) } \\
\mathrm{km} \mathrm{s}^{-1}\end{array}$ & $\begin{array}{r}\int T_{\mathrm{R}}^{*} \mathrm{~d} v \\
\mathrm{~K} \mathrm{~km} \mathrm{~s}^{-1}\end{array}$ \\
\hline \multirow[t]{3}{*}{ L1448 IRS3 } & $\mathrm{CH}_{3} \mathrm{OH}$ & $0.86(0.04)$ & $4.76(0.01)$ & $0.70(0.04)$ & $0.60(0.04)$ & $4.02(0.03)$ & $0.30(0.02)$ \\
\hline & $\mathrm{c}-\mathrm{C}_{3} \mathrm{H}_{2}$ & $0.99(0.08)$ & $4.63(0.03)$ & $0.47(0.03)$ & $0.46(0.04)$ & $3.92(0.02)$ & $0.15(0.03)$ \\
\hline & DCN & $0.76(0.03)$ & $3.99(0.01)$ & $0.34(0.06)$ & .. & .. &. \\
\hline \multirow[t]{3}{*}{ L1448NW } & $\mathrm{CH}_{3} \mathrm{OH}$ & $0.74(0.03)$ & $4.21(0.04)$ & $0.47(0.02)$ & $0.63(0.03)$ & $4.82(0.01)$ & $0.46(0.02)$ \\
\hline & $\mathrm{c}-\mathrm{C}_{3} \mathrm{H}_{2}$ & $0.70(0.05)$ & $4.63(0.02)$ & $0.29(0.02)$ & $0.50(0.03)$ & $3.98(0.01)$ & $0.24(0.02)$ \\
\hline & $\mathrm{DCN}$ & $0.83(0.03)$ & $3.95(0.01)$ & $0.43(0.06)$ & & & \\
\hline \multirow[t]{3}{*}{$\mathrm{L} 1448 \mathrm{~N}(+25,-35)$} & $\mathrm{CH}_{3} \mathrm{OH}$ & $4.14(0.30)$ & $4.37(0.28)$ & $0.96(0.09)$ & $0.79(0.06)$ & $4.58(0.02)$ & $0.56(0.04)$ \\
\hline & $\mathrm{c}-\mathrm{C}_{3} \mathrm{H}_{2}$ & $1.36(0.05)$ & $4.75(0.02)$ & $0.70(0.03)$ & $0.38(0.04)$ & $4.57(0.02)$ & $0.12(0.02)$ \\
\hline & DCN & $1.17(0.07)$ & $4.39(0.03)$ & $0.48(0.24)$ & & &. \\
\hline \multirow[t]{3}{*}{$\mathrm{L} 1448 \mathrm{~N}(+57,-110)$} & $\mathrm{CH}_{3} \mathrm{OH}$ & $1.54(0.21)$ & $4.78(0.24)$ & $0.33(0.07)$ & $0.54(0.10)$ & $4.65(0.02)$ & $0.18(0.04)$ \\
\hline & $\mathrm{c}-\mathrm{C}_{3} \mathrm{H}_{2}$ & $1.01(0.11)$ & $4.90(0.09)$ & $0.26(0.05)$ & $0.44(0.07)$ & $4.48(0.01)$ & $0.13(0.04)$ \\
\hline & $\mathrm{DCN}$ & $1.20(0.06)$ & $4.74(0.03)$ & $0.25(0.01)$ & .. &.. & .. \\
\hline \multirow[t]{3}{*}{ L1448 MMS } & $\mathrm{CH}_{3} \mathrm{OH}$ & $1.64(0.09)$ & $5.00(0.02)$ & $0.67(0.04)$ & .. & .. & .. \\
\hline & $\mathrm{c}-\mathrm{C}_{3} \mathrm{H}_{2}$ & $1.07(0.03)$ & $4.84(0.01)$ & $0.60(0.02)$ & .. & .. & .. \\
\hline & $\mathrm{DCN}$ & $0.96(0.05)$ & $4.78(0.02)$ & $0.66(0.30)$ & .. & .. & .. \\
\hline \multirow[t]{3}{*}{$\mathrm{L} 1448 \mathrm{~N}(33,-77)$} & $\mathrm{CH}_{3} \mathrm{OH}$ & $7.23(0.51)$ & $8.23(0.67)$ & $0.75(0.06)$ & $1.02(0.11)$ & $4.79(0.03)$ & $0.23(0.03)$ \\
\hline & $\mathrm{c}-\mathrm{C}_{3} \mathrm{H}_{2}$ & $1.19(0.13)$ & $4.91(0.04)$ & $0.27(0.02)$ & .. & .. & .. \\
\hline & $\mathrm{DCN}$ & $1.30(0.15)$ & $4.53(0.09)$ & $0.14(0.02)$ & .. & .. & .. \\
\hline \multirow[t]{4}{*}{ I03282 } & $\mathrm{CH}_{3} \mathrm{OH}$ & $1.00(0.05)$ & $7.33(0.01)$ & $0.33(0.02)$ & .. & .. & .. \\
\hline & $\mathrm{c}-\mathrm{C}_{3} \mathrm{H}_{2}$ & $0.72(0.06)$ & $6.94(0.02)$ & $0.13(0.01)$ & .. & .. & .. \\
\hline & $\mathrm{DCN}$ & $0.42(0.03)$ & $6.70(0.01)$ & $0.10(0.02)$ & .. & .. & .. \\
\hline & $\mathrm{C}^{18} \mathrm{O}$ & $0.95(0.03)$ & $7.48(0.01)$ & $0.89(0.02)$ & $0.51(0.19)$ & $8.48(0.06)$ & $0.05(0.02)$ \\
\hline \multirow[t]{3}{*}{ НH211 } & $\mathrm{CH}_{3} \mathrm{OH}$ & $4.05(0.71)$ & $8.57(0.26)$ & $0.25(0.05)$ & $0.69(0.02)$ & $9.03(0.03)$ & $0.51(0.02)$ \\
\hline & $\mathrm{c}-\mathrm{C}_{3} \mathrm{H}_{2}$ & $0.50(0.03)$ & $9.06(0.01)$ & $0.15(0.01)$ & .. & .. & .. \\
\hline & DCN & $0.48(0.03)$ & $8.83(0.01)$ & $0.11(0.02)$ & .. & .. & .. \\
\hline \multirow[t]{3}{*}{$\mathrm{HH} 211(-14,7)$} & $\mathrm{CH}_{3} \mathrm{OH}$ & $0.84(0.06)$ & $8.92(0.01)$ & $0.27(0.02)$ & .. & .. & .. \\
\hline & $\mathrm{c}-\mathrm{C}_{3} \mathrm{H}_{2}$ & $0.46(0.08)$ & $8.94(0.03)$ & $0.05(0.01)$ & .. & .. & .. \\
\hline & $\mathrm{DCN}$ & $0.70(0.15)$ & $8.73(0.05)$ & $0.09(0.01)$ & .. & .. & .. \\
\hline \multirow[t]{3}{*}{ HH211(18, -9) } & $\mathrm{CH}_{3} \mathrm{OH}$ & $0.78(0.03)$ & $9.07(0.01)$ & $0.44(0.02)$ & .. & .. & .. \\
\hline & $\mathrm{c}-\mathrm{C}_{3} \mathrm{H}_{2}$ & $0.45(0.04)$ & $9.08(0.02)$ & $0.13(0.01)$ & .. & .. & .. \\
\hline & $\mathrm{DCN}$ & $0.34(0.03)$ & $8.91(0.02)$ & $0.04(0.01)$ & & & .. \\
\hline \multirow[t]{2}{*}{$\mathrm{HH} 211(41,14)$} & $\mathrm{CH}_{3} \mathrm{OH}$ & $1.17(0.12)$ & $8.90(0.25)$ & $0.31(0.06)$ & $0.60(0.18)$ & $9.19(0.05)$ & $0.11(0.05)$ \\
\hline & $\mathrm{c}-\mathrm{C}_{3} \mathrm{H}_{2}$ & $0.94(0.14)$ & $8.94(0.08)$ & $0.10(0.02)$ & $0.18(0.08)$ & $9.23(0.03)$ & $0.03(0.01)$ \\
\hline \multirow[t]{2}{*}{ HH211(41, -14) } & $\mathrm{CH}_{3} \mathrm{OH}$ & $1.55(0.07)$ & $8.77(0.02)$ & $0.45(0.02)$ & .. &.. & .. \\
\hline & $\mathrm{c}-\mathrm{C}_{3} \mathrm{H}_{2}$ & $0.60(0.15)$ & $9.09(0.06)$ & $0.04(0.01)$ & .. & .. & .. \\
\hline \multirow[t]{2}{*}{ HH211 $(-43,14)$} & $\mathrm{CH}_{3} \mathrm{OH}$ & $0.90(0.08)$ & $8.90(0.02)$ & $0.23(0.02)$ & .. & .. & .. \\
\hline & $\mathrm{c}-\mathrm{C}_{3} \mathrm{H}_{2}$ & $0.59(0.10)$ & $9.11(0.06)$ & $0.04(0.01)$ & .. & .. & .. \\
\hline \multirow[t]{4}{*}{ IRAM 04191} & $\mathrm{CH}_{3} \mathrm{OH}$ & $0.63(0.02)$ & $6.65(0.01)$ & $0.38(0.01)$ & & 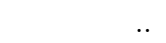 & .. \\
\hline & $\mathrm{c}-\mathrm{C}_{3} \mathrm{H}_{2}$ & $0.41(0.02)$ & $6.50(0.01)$ & $0.15(0.01)$ & $0.24(0.06)$ & $7.01(0.03)$ & $0.02(0.01)$ \\
\hline & $\mathrm{DCN}$ & $0.49(0.04)$ & $6.60(0.02)$ & $0.04(0.01)$ & .. & .. & .. \\
\hline & $\mathrm{C}^{18} \mathrm{O}$ & $0.72(0.02)$ & $6.71(0.02)$ & $1.15(0.06)$ & $0.33(0.07)$ & $6.49(0.03)$ & $0.12(0.06)$ \\
\hline \multirow[t]{2}{*}{ IRAM $04191(6,17)$} & $\mathrm{CH}_{3} \mathrm{OH}$ & $0.59(0.02)$ & $6.94(0.01)$ & $0.39(0.02)$ & .. & .. & .. \\
\hline & $\mathrm{c}-\mathrm{C}_{3} \mathrm{H}_{2}$ & $0.29(0.05)$ & $6.86(0.02)$ & $0.05(0.01)$ & .. & .. & .. \\
\hline \multirow[t]{2}{*}{ Haro6-10 } & $\mathrm{CH}_{3} \mathrm{OH}$ & $0.37(0.04)$ & $6.35(0.01)$ & $0.12(0.01)$ & .. & .. & .. \\
\hline & $\mathrm{c}-\mathrm{C}_{3} \mathrm{H}_{2}$ & $0.39(0.02)$ & $6.29(0.01)$ & $0.14(0.01)$ & .. & .. & .. \\
\hline L1551IRS5 & $\mathrm{CH}_{3} \mathrm{OH}$ & $1.06(0.02)$ & $6.60(0.01)$ & $0.62(0.01)$ & &.. & .. \\
\hline & $\mathrm{c}-\mathrm{C}_{3} \mathrm{H}_{2}$ & $1.14(0.22)$ & $6.80(0.01)$ & $0.09(0.02)$ & $0.66(0.03)$ & $6.39(0.02)$ & $0.29(0.02)$ \\
\hline & $\mathrm{DCN}$ & $0.71(0.04)$ & $6.09(0.01)$ & $0.26(0.09)$ & .. &.. & \\
\hline & $\mathrm{C}^{18} \mathrm{O}$ & $1.07(0.11)$ & $6.01(0.12)$ & $0.35(0.09)$ & $0.61(0.03)$ & $6.48(0.01)$ & $0.78(0.09)$ \\
\hline L1551NE & $\mathrm{CH}_{3} \mathrm{OH}$ & $2.09(0.17)$ & $6.83(0.04)$ & $0.39(0.03)$ & .. & .. & .. \\
\hline & $\mathrm{c}-\mathrm{C}_{3} \mathrm{H}_{2}$ & $0.58(0.04)$ & $6.61(0.02)$ & $0.15(0.01)$ & .. & .. & .. \\
\hline & $\mathrm{C}^{18} \mathrm{O}$ & $0.67(0.04)$ & $6.63(0.02)$ & $0.82(0.02)$ & $0.43(0.01)$ & $6.78(0.01)$ & $0.66(0.02)$ \\
\hline $\operatorname{L} 1551 \mathrm{NE}(-168,-96)$ & $\mathrm{CH}_{3} \mathrm{OH}$ & $0.69(0.09)$ & $6.39(0.16)$ & $0.22(0.03)$ & $0.66(0.16)$ & $6.97(0.04)$ & $0.11(0.03)$ \\
\hline & $\mathrm{c}-\mathrm{C}_{3} \mathrm{H}_{2}$ & $0.62(0.10)$ & $6.33(0.05)$ & $0.10(0.01)$ &.. & .. & .. \\
\hline L1535 & $\mathrm{CH}_{3} \mathrm{OH}$ & $0.61(0.07)$ & $5.90(0.01)$ & $0.13(0.02)$ & .. & .. & .. \\
\hline & $\mathrm{c}-\mathrm{C}_{3} \mathrm{H}_{2}$ & $0.70(0.07)$ & $5.76(0.03)$ & $0.19(0.02)$ & $0.23(0.04)$ & $5.71(0.01)$ & $0.08(0.02)$ \\
\hline & $\mathrm{DCN}$ & $0.53(0.14)$ & $5.47(0.05)$ & $0.08(0.02)$ & .. &.. & .. \\
\hline & $\mathrm{C}^{18} \mathrm{O}$ & $0.72(0.02)$ & $5.82(0.01)$ & $1.03(0.03)$ & .. & .. & .. \\
\hline
\end{tabular}


Table 3. continued.

\begin{tabular}{|c|c|c|c|c|c|c|c|}
\hline Source & Molecule & $\begin{array}{r}\Delta v \\
\mathrm{~km} \mathrm{~s}^{-1}\end{array}$ & $\begin{array}{r}v \text { (Broad/Single) } \\
\mathrm{km} \mathrm{s}^{-1}\end{array}$ & $\begin{array}{r}\int T_{\mathrm{R}}^{*} \mathrm{~d} v \\
\mathrm{~K} \mathrm{~km} \mathrm{~s}^{-1}\end{array}$ & $\begin{array}{r}\Delta v \\
\mathrm{~km} \mathrm{~s}^{-1}\end{array}$ & $\begin{array}{r}v \text { (Narrow) } \\
\mathrm{km} \mathrm{s}^{-1}\end{array}$ & $\begin{array}{r}\int T_{\mathrm{R}}^{*} \mathrm{~d} v \\
\mathrm{~K} \mathrm{~km} \mathrm{~s}^{-1}\end{array}$ \\
\hline \multirow[t]{4}{*}{ TMR1 } & $\mathrm{CH}_{3} \mathrm{OH}$ & $0.55(0.10)$ & $6.54(0.02)$ & $0.08(0.02)$ & .. & .. & .. \\
\hline & $\mathrm{c}-\mathrm{C}_{3} \mathrm{H}_{2}$ & $0.53(0.04)$ & $6.46(0.02)$ & $0.11(0.01)$ & .. & .. & .. \\
\hline & DCN & $0.24(0.06)$ & $5.35(0.03)$ & $0.04(0.01)$ & .. &.. & .. \\
\hline & $\mathrm{C}^{18} \mathrm{O}$ & $0.58(0.01)$ & $6.52(0.01)$ & $1.39(0.02)$ & $0.40(0.02)$ & $5.78(0.01)$ & $0.32(0.02)$ \\
\hline \multirow[t]{3}{*}{ L1527 } & $\mathrm{CH}_{3} \mathrm{OH}$ & $0.42(0.02)$ & $5.90(0.01)$ & $0.31(0.01)$ & & .. & .. \\
\hline & c- $\mathrm{C}_{3} \mathrm{H}_{2}$ & $0.54(0.02)$ & $5.81(0.01)$ & $0.38(0.01)$ & $0.24(0.02)$ & $5.77(0.01)$ & $0.07(0.01)$ \\
\hline & $\mathrm{C}^{18} \mathrm{O}$ & $0.51(0.01)$ & $6.00(0.01)$ & $1.25(0.02)$ & .. & .. & .. \\
\hline \multirow{3}{*}{ RNO43 } & $\mathrm{CH}_{3} \mathrm{OH}$ & $0.56(0.04)$ & $10.30(0.01)$ & $0.14(0.01)$ & .. & .. & .. \\
\hline & $\mathrm{c}-\mathrm{C}_{3} \mathrm{H}_{2}$ & $0.86(0.05)$ & $10.24(0.03)$ & $0.13(0.01)$ & .. & .. & .. \\
\hline & DCN & $0.77(0.07)$ & $10.13(0.03)$ & $0.14(0.01)$ & 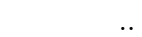 & & - \\
\hline \multirow[t]{4}{*}{ HH25 } & $\mathrm{CH}_{3} \mathrm{OH}$ & $3.58(0.20)$ & $10.02(0.62)$ & $0.54(0.04)$ & $0.99(0.05)$ & $10.50(0.01)$ & $0.35(0.02)$ \\
\hline & $\mathrm{c}-\mathrm{C}_{3} \mathrm{H}_{2}$ & $0.53(0.05)$ & $10.41(0.02)$ & $0.08(0.01)$ & .. & .. & .. \\
\hline & $\mathrm{DCN}$ & $0.85(0.15)$ & $10.03(0.05)$ & $0.06(0.01)$ & .. &.. & .. \\
\hline & $\mathrm{C}^{18} \mathrm{O}$ & $0.64(0.03)$ & $10.54(0.01)$ & $0.53(0.02)$ & .. & .. & .. \\
\hline HH25 $(224,30)$ & $\mathrm{CH}_{3} \mathrm{OH}$ & $4.58(0.39)$ & 10.34(0.09) & $0.29(0.03)$ & .. & .. & .. \\
\hline \multirow[t]{3}{*}{ HH111 } & $\mathrm{CH}_{3} \mathrm{OH}$ & $0.72(0.04)$ & $8.75(0.01)$ & $0.32(0.02)$ & .. &.. & .. \\
\hline & $\mathrm{c}-\mathrm{C}_{3} \mathrm{H}_{2}$ & $1.12(0.16)$ & $8.79(0.07)$ & $0.11(0.01)$ & .. & .. & .. \\
\hline & $\mathrm{C}^{18} \mathrm{O}$ & $0.83(0.01)$ & $8.76(0.01)$ & $1.99(0.02)$ & .. &.. & .. \\
\hline \multirow{4}{*}{ VLA1623 } & $\mathrm{CH}_{3} \mathrm{OH}$ & $1.65(0.07)$ & $3.47(0.01)$ & $0.70(0.03)$ & .. & .. & .. \\
\hline & $\mathrm{c}-\mathrm{C}_{3} \mathrm{H}_{2}$ & $0.70(0.03)$ & $3.61(0.01)$ & $0.25(0.01)$ & .. & . & 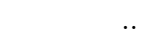 \\
\hline & DCN & $0.85(0.03)$ & $3.53(0.01)$ & $0.54(0.02)$ & .. & .. & .. \\
\hline & $\mathrm{C}^{18} \mathrm{O}$ & $0.95(0.01)$ & $3.61(0.01)$ & $6.03(0.06)$ & $0.72(0.04)$ & $2.54(0.02)$ & $1.08(0.06)$ \\
\hline \multirow{3}{*}{ VLA1623(-42, 16) } & $\mathrm{CH}_{3} \mathrm{OH}$ & $1.86(0.05)$ & $3.00(0.02)$ & $0.89(0.04)$ & $0.79(0.11)$ & $2.63(0.02)$ & $0.11(0.02)$ \\
\hline & $\mathrm{c}-\mathrm{C}_{3} \mathrm{H}_{2}$ & $1.25(0.18)$ & $3.62(0.10)$ & $0.07(0.01)$ & .. & .. & .. \\
\hline & $\mathrm{DCN}$ & $1.49(0.10)$ & $3.27(0.05)$ & $0.15(0.01)$ & & & \\
\hline \multirow[t]{3}{*}{$\operatorname{VLA} 1623(45,-24)$} & $\mathrm{CH}_{3} \mathrm{OH}$ & $1.41(0.38)$ & $3.34(0.74)$ & $0.48(0.28)$ & $0.84(0.45)$ & $3.46(0.08)$ & $0.18(0.17)$ \\
\hline & c- $\mathrm{C}_{3} \mathrm{H}_{2}$ & $0.66(0.06)$ & $3.58(0.03)$ & $0.15(0.01)$ & .. & .. & .. \\
\hline & $\mathrm{DCN}$ & $0.82(0.04)$ & $3.19(0.02)$ & $0.37(0.02)$ & .. &.. & .. \\
\hline \multirow[t]{3}{*}{ VLA1623(16, 76) } & $\mathrm{CH}_{3} \mathrm{OH}$ & $1.61(0.10)$ & $3.42(0.04)$ & $1.22(0.09)$ & $0.92(0.14)$ & $2.63(0.02)$ & $0.25(0.06)$ \\
\hline & $\mathrm{c}-\mathrm{C}_{3} \mathrm{H}_{2}$ & $0.71(0.04)$ & $3.42(0.01)$ & $0.27(0.01)$ & .. & & .. \\
\hline & DCN & $1.30(0.04)$ & $3.04(0.02)$ & $0.41(0.01)$ & .. & .. & .. \\
\hline \multirow[t]{3}{*}{ VLA1623(16, 31) } & $\mathrm{CH}_{3} \mathrm{OH}$ & $1.64(0.11)$ & $3.17(0.13)$ & $0.57(0.05)$ & $0.57(0.07)$ & $3.61(0.02)$ & $0.22(0.03)$ \\
\hline & $\mathrm{c}-\mathrm{C}_{3} \mathrm{H}_{2}$ & $0.67(0.03)$ & $3.58(0.01)$ & $0.35(0.01)$ & .. & .. & .. \\
\hline & DCN & $0.67(0.02)$ & $3.52(0.01)$ & $0.46(0.02)$ & 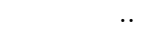 & .. & .. \\
\hline \multirow[t]{3}{*}{ VLA1623(45, 6) } & $\mathrm{CH}_{3} \mathrm{OH}$ & $0.96(0.34)$ & $3.25(0.46)$ & $0.21(0.65)$ & $0.78(0.33)$ & $3.43(0.11)$ & $0.45(0.62)$ \\
\hline & $\mathrm{c}-\mathrm{C}_{3} \mathrm{H}_{2}$ & $0.54(0.03)$ & $3.53(0.01)$ & $0.22(0.01)$ & .. & .. & .. \\
\hline & $\mathrm{DCN}$ & $0.53(0.03)$ & $3.32(0.01)$ & $0.54(0.27)$ & .. &. & .. \\
\hline \multirow[t]{2}{*}{ VLA1623(-71, 36) } & $\mathrm{CH}_{3} \mathrm{OH}$ & $2.24(0.34)$ & $3.06(0.24)$ & $0.68(0.18)$ & $1.03(0.30)$ & $2.75(0.08)$ & $0.33(0.12)$ \\
\hline & $\mathrm{c}-\mathrm{C}_{3} \mathrm{H}_{2}$ & $0.48(0.10)$ & $3.48(0.04)$ & $0.10(0.02)$ & .. & 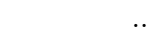 & .. \\
\hline LFAM1 & $\mathrm{CH}_{3} \mathrm{OH}$ & $2.21(0.33)$ & $3.70(0.46)$ & $0.67(0.19)$ & $1.36(0.22)$ & $3.33(0.05)$ & $0.35(0.12)$ \\
\hline EL29 & $\mathrm{CH}_{3} \mathrm{OH}$ & $1.32(0.44)$ & $3.97(0.09)$ & $0.07(0.03)$ & .. & .. & .. \\
\hline \multirow[t]{2}{*}{ I16244 } & $\mathrm{CH}_{3} \mathrm{OH}$ & $1.23(0.12)$ & $3.82(0.03)$ & $0.24(0.03)$ & .. &. & .. \\
\hline & $\mathrm{C}^{18} \mathrm{O}$ & $1.63(0.02)$ & $3.48(0.01)$ & $5.91(0.08)$ & .. &.. & .. \\
\hline $\mathrm{I} 16244(35,14)$ & $\mathrm{CH}_{3} \mathrm{OH}$ & $1.87(0.27)$ & $3.84(0.06)$ & $0.20(0.03)$ & .. & .. & .. \\
\hline \multirow[t]{4}{*}{ I16293 } & $\mathrm{CH}_{3} \mathrm{OH}$ & $4.49(0.19)$ & $4.00(0.04)$ & $1.16(0.06)$ & $0.97(0.04)$ & $3.86(0.01)$ & $0.64(0.03)$ \\
\hline & $\mathrm{c}-\mathrm{C}_{3} \mathrm{H}_{2}$ & $1.50(0.05)$ & $4.10(0.02)$ & $0.41(0.01)$ & $0.28(0.02)$ & $3.93(0.01)$ & $0.07(0.01)$ \\
\hline & DCN & $1.90(0.08)$ & $3.88(0.03)$ & $0.65(0.13)$ & .. & .. & .. \\
\hline & $\mathrm{C}^{18} \mathrm{O}$ & $1.48(0.07)$ & $3.85(0.02)$ & $3.46(0.17)$ & $0.54(0.04)$ & $3.91(0.01)$ & $1.42(0.19)$ \\
\hline $\mathrm{I} 16293(60,-45)$ & $\mathrm{CH}_{3} \mathrm{OH}$ & $2.90(0.11)$ & $1.95(0.03)$ & $1.11(0.05)$ & $0.81(0.02)$ & $3.76(0.01)$ & $0.90(0.02)$ \\
\hline & $\mathrm{c}-\mathrm{C}_{3} \mathrm{H}_{2}$ & $0.78(0.05)$ & $3.87(0.02)$ & $0.31(0.01)$ & $0.16(0.04)$ & $3.83(0.02)$ & $0.03(0.01)$ \\
\hline & $\mathrm{DCN}$ & $0.73(0.02)$ & $3.65(0.01)$ & $0.45(0.08)$ & .. & 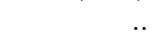 & .. \\
\hline L483 & $\mathrm{CH}_{3} \mathrm{OH}$ & $0.83(0.09)$ & $5.57(0.07)$ & $0.19(0.04)$ & $0.43(0.05)$ & $5.29(0.05)$ & $0.19(0.03)$ \\
\hline & $c-\mathrm{C}_{3} \mathrm{H}_{2}$ & $0.77(0.07)$ & $5.37(0.05)$ & $0.11(0.03)$ & $0.38(0.03)$ & $5.27(0.01)$ & $0.16(0.03)$ \\
\hline & $\mathrm{DCN}$ & $0.29(0.03)$ & $5.15(0.01)$ & $0.06(0.02)$ & .. &.. & .. \\
\hline $\mathrm{L} 483(-30,5)$ & $\mathrm{CH}_{3} \mathrm{OH}$ & $1.13(0.13)$ & $5.31(0.03)$ & $0.26(0.04)$ & $0.35(0.02)$ & $5.26(0.04)$ & $0.24(0.02)$ \\
\hline & $c-\mathrm{C}_{3} \mathrm{H}_{2}$ & $0.51(0.22)$ & $4.91(0.11)$ & $0.04(0.02)$ & $0.40(0.01)$ & $5.26(0.01)$ & $0.35(0.02)$ \\
\hline & $\mathrm{DCN}$ & $0.44(0.02)$ & $5.15(0.01)$ & $0.13(0.02)$ & &.. & .. \\
\hline $\mathrm{L} 483(40,-5)$ & $\mathrm{CH}_{3} \mathrm{OH}$ & $0.66(0.31)$ & $5.39(0.16)$ & $0.21(0.29)$ & $0.48(0.76)$ & $5.40(0.04)$ & $0.04(0.20)$ \\
\hline & $\mathrm{c}-\mathrm{C}_{3} \mathrm{H}_{2}$ & $0.61(0.04)$ & $5.35(0.01)$ & $0.15(0.01)$ & .. & .. & .. \\
\hline & $\mathrm{DCN}$ & $0.67(0.09)$ & $5.37(0.04)$ & $0.08(0.01)$ & .. & .. & .. \\
\hline L483SW $(0,0)$ & $\mathrm{CH}_{3} \mathrm{OH}$ & $0.51(0.03)$ & $5.43(0.01)$ & $0.20(0.01)$ & .. & .. & .. \\
\hline & $\mathrm{c}-\mathrm{C}_{3} \mathrm{H}_{2}$ & $0.38(0.03)$ & $5.30(0.01)$ & $0.08(0.01)$ & .. & .. & .. \\
\hline
\end{tabular}


Table 3. continued.

\begin{tabular}{|c|c|c|c|c|c|c|c|}
\hline Source & Molecule & $\begin{array}{r}\Delta v \\
\mathrm{~km} \mathrm{~s}^{-1}\end{array}$ & $\begin{array}{r}v(\text { Broad/Single }) \\
\mathrm{km} \mathrm{s}^{-1}\end{array}$ & $\begin{array}{r}\int T_{\mathrm{R}}^{*} \mathrm{~d} v \\
\mathrm{~K} \mathrm{~km} \mathrm{~s}^{-1}\end{array}$ & $\begin{array}{r}\Delta v \\
\mathrm{~km} \mathrm{~s}^{-1}\end{array}$ & $\begin{array}{r}v \text { (Narrow) } \\
\mathrm{km} \mathrm{s}^{-1}\end{array}$ & $\begin{array}{r}\int T_{\mathrm{R}}^{*} \mathrm{~d} v \\
\mathrm{~K} \mathrm{~km} \mathrm{~s}^{-1}\end{array}$ \\
\hline \multirow[t]{4}{*}{ S68N } & $\mathrm{CH}_{3} \mathrm{OH}$ & $6.70(0.08)$ & $8.91(0.02)$ & $3.41(0.05)$ & $1.63(0.03)$ & $8.67(0.01)$ & $1.16(0.03)$ \\
\hline & $\mathrm{c}-\mathrm{C}_{3} \mathrm{H}_{2}$ & $1.11(0.05)$ & $8.30(0.02)$ & $0.28(0.01)$ & .. & .. & .. \\
\hline & $\mathrm{DCN}$ & $1.41(0.07)$ & $8.34(0.03)$ & $0.22(0.06)$ & .. & .. & .. \\
\hline & $\mathrm{C}^{18} \mathrm{O}$ & $1.25(0.01)$ & $8.47(0.01)$ & $5.13(0.04)$ & $0.63(0.07)$ & $6.93(0.03)$ & $0.32(0.03)$ \\
\hline \multirow[t]{3}{*}{ SMM1 } & $\mathrm{CH}_{3} \mathrm{OH}$ & $5.48(0.33)$ & $4.70(0.67)$ & $1.83(0.13)$ & $2.56(0.16)$ & $7.71(0.04)$ & $1.19(0.10)$ \\
\hline & $\mathrm{c}-\mathrm{C}_{3} \mathrm{H}_{2}$ & $0.93(0.22)$ & $7.87(0.08)$ & $0.09(0.01)$ & .. &.. & .. \\
\hline & $\mathrm{DCN}$ & $0.43(0.09)$ & $7.63(0.03)$ & $0.08(0.01)$ & .. & .. & .. \\
\hline \multirow[t]{3}{*}{ SMM4 } & $\mathrm{CH}_{3} \mathrm{OH}$ & $8.29(0.48)$ & $8.26(0.10)$ & $1.48(0.11)$ & $1.96(0.07)$ & $8.07(0.01)$ & $1.28(0.05)$ \\
\hline & $c-\mathrm{C}_{3} \mathrm{H}_{2}$ & $1.19(0.07)$ & $8.15(0.03)$ & $0.34(0.02)$ & $0.91(0.08)$ & $6.83(0.04)$ & $0.15(0.01)$ \\
\hline & DCN & $2.04(0.13)$ & $7.54(0.06)$ & $0.20(0.07)$ & .. & & .. \\
\hline \multirow[t]{2}{*}{$\mathrm{S} 68 \mathrm{~N}(46,66)$} & $\mathrm{CH}_{3} \mathrm{OH}$ & $5.02(0.53)$ & $7.06(0.18)$ & $0.55(0.09)$ & $2.00(0.18)$ & $8.23(0.03)$ & $0.59(0.06)$ \\
\hline & $\mathrm{c}-\mathrm{C}_{3} \mathrm{H}_{2}$ & $0.93(0.12)$ & $7.95(0.04)$ & $0.15(0.01)$ & & & \\
\hline \multirow[t]{3}{*}{ B335 } & $\mathrm{CH}_{3} \mathrm{OH}$ & $0.61(0.11)$ & $8.38(0.29)$ & $0.12(0.03)$ & $0.25(0.08)$ & $8.34(0.01)$ & $0.03(0.01)$ \\
\hline & $\mathrm{c}-\mathrm{C}_{3} \mathrm{H}_{2}$ & $0.44(0.02)$ & $8.31(0.01)$ & $0.09(0.01)$ & .. & .. & .. \\
\hline & $\mathrm{DCN}$ & $0.39(0.05)$ & $8.08(0.03)$ & $0.01(0.01)$ & & .. & .. \\
\hline \multirow[t]{3}{*}{ B335 $(-60,24)$} & $\mathrm{CH}_{3} \mathrm{OH}$ & $0.53(0.07)$ & $8.34(0.04)$ & $0.12(0.02)$ & $0.19(0.04)$ & $8.29(0.01)$ & $0.03(0.01)$ \\
\hline & $\mathrm{c}-\mathrm{C}_{3} \mathrm{H}_{2}$ & $0.46(0.03)$ & $8.31(0.01)$ & $0.10(0.01)$ & .. & .. & .. \\
\hline & $\mathrm{DCN}$ & $0.19(0.04)$ & $8.06(0.02)$ & $0.02(0.01)$ & .. & .. & .. \\
\hline \multirow[t]{4}{*}{ I20126 } & $\mathrm{CH}_{3} \mathrm{OH}$ & $4.55(0.33)$ & $-3.00(0.08)$ & $0.68(0.06)$ & .. & .. & .. \\
\hline & $\mathrm{c}-\mathrm{C}_{3} \mathrm{H}_{2}$ & $1.82(0.22)$ & $-3.76(0.08)$ & $0.38(0.03)$ & .. & .. & .. \\
\hline & DCN & $2.11(0.15)$ & $-3.87(0.08)$ & $0.52(0.04)$ & .. & .. & .. \\
\hline & $\mathrm{C}^{18} \mathrm{O}$ & $2.57(0.08)$ & $-3.69(0.04)$ & $2.25(0.06)$ & $0.69(0.15)$ & $-3.13(0.08)$ & $0.14(0.05)$ \\
\hline \multirow[t]{4}{*}{ L1152 } & $\mathrm{CH}_{3} \mathrm{OH}$ & $0.70(0.08)$ & $2.61(0.22)$ & $0.12(0.02)$ & $0.25(0.13)$ & $2.51(0.02)$ & $0.01(0.01)$ \\
\hline & $\mathrm{c}-\mathrm{C}_{3} \mathrm{H}_{2}$ & $0.97(0.14)$ & $2.77(0.06)$ & $0.06(0.01)$ & $0.48(0.02)$ & $2.68(0.01)$ & $0.15(0.01)$ \\
\hline & $\mathrm{DCN}$ & $0.36(0.05)$ & $2.48(0.02)$ & $0.05(0.02)$ & & & \\
\hline & $\mathrm{C}^{18} \mathrm{O}$ & $0.77(0.05)$ & $2.69(0.04)$ & $0.71(0.08)$ & $0.33(0.09)$ & $2.44(0.04)$ & $0.13(0.08)$ \\
\hline \multirow[t]{3}{*}{$\mathrm{L} 1152(15,30)$} & $\mathrm{CH}_{3} \mathrm{OH}$ & $0.41(0.04)$ & $2.57(0.01)$ & $0.09(0.01)$ & & , & .. \\
\hline & $\mathrm{c}-\mathrm{C}_{3} \mathrm{H}_{2}$ & $0.33(0.03)$ & $2.55(0.01)$ & $0.08(0.01)$ & $0.20(0.05)$ & $2.89(0.02)$ & $0.02(0.01)$ \\
\hline & $\mathrm{DCN}$ & $0.79(0.13)$ & $2.42(0.06)$ & $0.06(0.01)$ & & & .. \\
\hline \multirow[t]{3}{*}{ L1157 } & $\mathrm{CH}_{3} \mathrm{OH}$ & $6.75(0.28)$ & $0.91(0.41)$ & $2.52(0.13)$ & $1.25(0.25)$ & $2.69(0.07)$ & $0.27(0.06)$ \\
\hline & $\mathrm{DCN}$ & $2.56(0.61)$ & $2.68(0.29)$ & $0.24(0.05)$ & & & \\
\hline & $\mathrm{C}^{18} \mathrm{O}$ & $0.45(0.04)$ & $2.74(0.03)$ & $0.58(0.08)$ & $0.37(0.14)$ & $2.32(0.08)$ & $0.14(0.08)$ \\
\hline $\operatorname{L} 1157(20,-60)$ & $\mathrm{CH}_{3} \mathrm{OH}$ & $6.20(0.19)$ & $-0.66(0.15)$ & $4.89(0.28)$ & $3.67(0.29)$ & $1.56(0.03)$ & $2.15(0.27)$ \\
\hline \multirow[t]{4}{*}{ L1174 } & $\mathrm{CH}_{3} \mathrm{OH}$ & $1.01(0.18)$ & $3.08(0.04)$ & $0.14(0.03)$ & .. & .. & .. \\
\hline & $\mathrm{c}-\mathrm{C}_{3} \mathrm{H}_{2}$ & $0.45(0.03)$ & $3.03(0.01)$ & $0.23(0.01)$ & .. & .. & .. \\
\hline & DCN & $1.05(0.18)$ & $2.65(0.06)$ & $0.14(0.02)$ & .. & .. & .. \\
\hline & $\mathrm{C}^{18} \mathrm{O}$ & $1.05(0.28)$ & $2.29(0.23)$ & $0.32(0.16)$ & $0.73(0.03)$ & $2.96(0.02)$ & $1.10(0.16)$ \\
\hline \multirow[t]{4}{*}{ L1251B } & $\mathrm{CH}_{3} \mathrm{OH}$ & $1.23(0.09)$ & $-3.78(0.02)$ & $0.51(0.04)$ & $0.46(0.15)$ & $-4.61(0.50)$ & $0.05(0.02)$ \\
\hline & $\mathrm{c}-\mathrm{C}_{3} \mathrm{H}_{2}$ & $1.01(0.03)$ & $-3.82(0.01)$ & $0.32(0.01)$ & .. & .. & .. \\
\hline & $\mathrm{DCN}$ & $0.84(0.06)$ & $-4.01(0.03)$ & $0.13(0.05)$ & - & .. & .. \\
\hline & $\mathrm{C}^{18} \mathrm{O}$ & $0.70(0.05)$ & $-3.52(0.03)$ & $1.17(0.09)$ & $0.34(0.07)$ & $-3.99(0.03)$ & $0.22(0.08)$ \\
\hline $\mathrm{L} 1251 \mathrm{~B}(128,-50)$ & $\mathrm{CH}_{3} \mathrm{OH}$ & $1.78(0.11)$ & $-3.92(0.02)$ & $0.36(0.03)$ & .. & .. & .. \\
\hline \multirow[t]{2}{*}{$\operatorname{L} 1251 \mathrm{~B}(-81,85)$} & $\mathrm{CH}_{3} \mathrm{OH}$ & $1.43(0.22)$ & $-3.55(0.05)$ & $0.13(0.02)$ & .. & .. & .. \\
\hline & $\mathrm{c}-\mathrm{C}_{3} \mathrm{H}_{2}$ & $0.90(0.13)$ & $-3.71(0.06)$ & $0.09(0.01)$ & - & .. & .. \\
\hline \multirow[t]{4}{*}{ CEPE } & $\mathrm{CH}_{3} \mathrm{OH}$ & $6.22(0.36)$ & $-12.00(0.87)$ & $0.83(0.07)$ & $2.43(0.22)$ & $-11.40(0.04)$ & $0.37(0.04)$ \\
\hline & $\mathrm{c}-\mathrm{C}_{3} \mathrm{H}_{2}$ & $1.01(0.13)$ & $-11.13(0.09)$ & $0.04(0.01)$ & & & \\
\hline & $\mathrm{DCN}$ & $0.87(0.09)$ & $-11.37(0.03)$ & $0.11(0.01)$ & .. & & 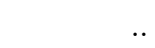 \\
\hline & $\mathrm{C}^{18} \mathrm{O}$ & $0.74(0.06)$ & $-11.01(0.03)$ & $0.90(0.07)$ & $0.44(0.11)$ & $-11.64(0.08)$ & $0.13(0.06)$ \\
\hline \multirow[t]{2}{*}{$\operatorname{CEPE}(4,17)$} & $\mathrm{CH}_{3} \mathrm{OH}$ & $5.77(0.26)$ & $-12.07(0.74)$ & $0.72(0.04)$ & $1.13(0.10)$ & $-11.08(0.03)$ & $0.20(0.02)$ \\
\hline & $\mathrm{c}-\mathrm{C}_{3} \mathrm{H}_{2}$ & $0.78(0.09)$ & $-11.14(0.04)$ & $0.07(0.01)$ & .. & & \\
\hline \multirow{3}{*}{$\operatorname{CEPE}(-24,-22)$} & $\mathrm{CH}_{3} \mathrm{OH}$ & $9.24(0.72)$ & $-12.43(0.88)$ & $0.64(0.06)$ & $0.92(0.14)$ & $-12.06(0.04)$ & $0.08(0.01)$ \\
\hline & $\mathrm{c}-\mathrm{C}_{3} \mathrm{H}_{2}$ & $0.42(0.11)$ & $-10.98(0.05)$ & $0.02(0.01)$ & .. & .. & .. \\
\hline & $\mathrm{DCN}$ & $1.38(0.14)$ & $-11.27(0.05)$ & $0.18(0.01)$ & & & \\
\hline L1262 & $\mathrm{CH}_{3} \mathrm{OH}$ & $1.01(0.11)$ & $4.21(0.17)$ & $0.18(0.02)$ & $0.32(0.03)$ & $4.22(0.01)$ & $0.11(0.01)$ \\
\hline & $\mathrm{c}-\mathrm{C}_{3} \mathrm{H}_{2}$ & $0.65(0.04)$ & $4.05(0.01)$ & $0.15(0.01)$ & $0.21(0.07)$ & $4.08(0.02)$ & $0.02(0.01)$ \\
\hline & $\mathrm{DCN}$ & $0.47(0.05)$ & $3.90(0.02)$ & $0.04(0.01)$ & & & .. \\
\hline & $\mathrm{C}^{18} \mathrm{O}$ & $0.58(0.02)$ & $4.09(0.01)$ & $0.98(0.03)$ & $0.22(0.05)$ & $3.50(0.02)$ & $0.08(0.02)$ \\
\hline
\end{tabular}



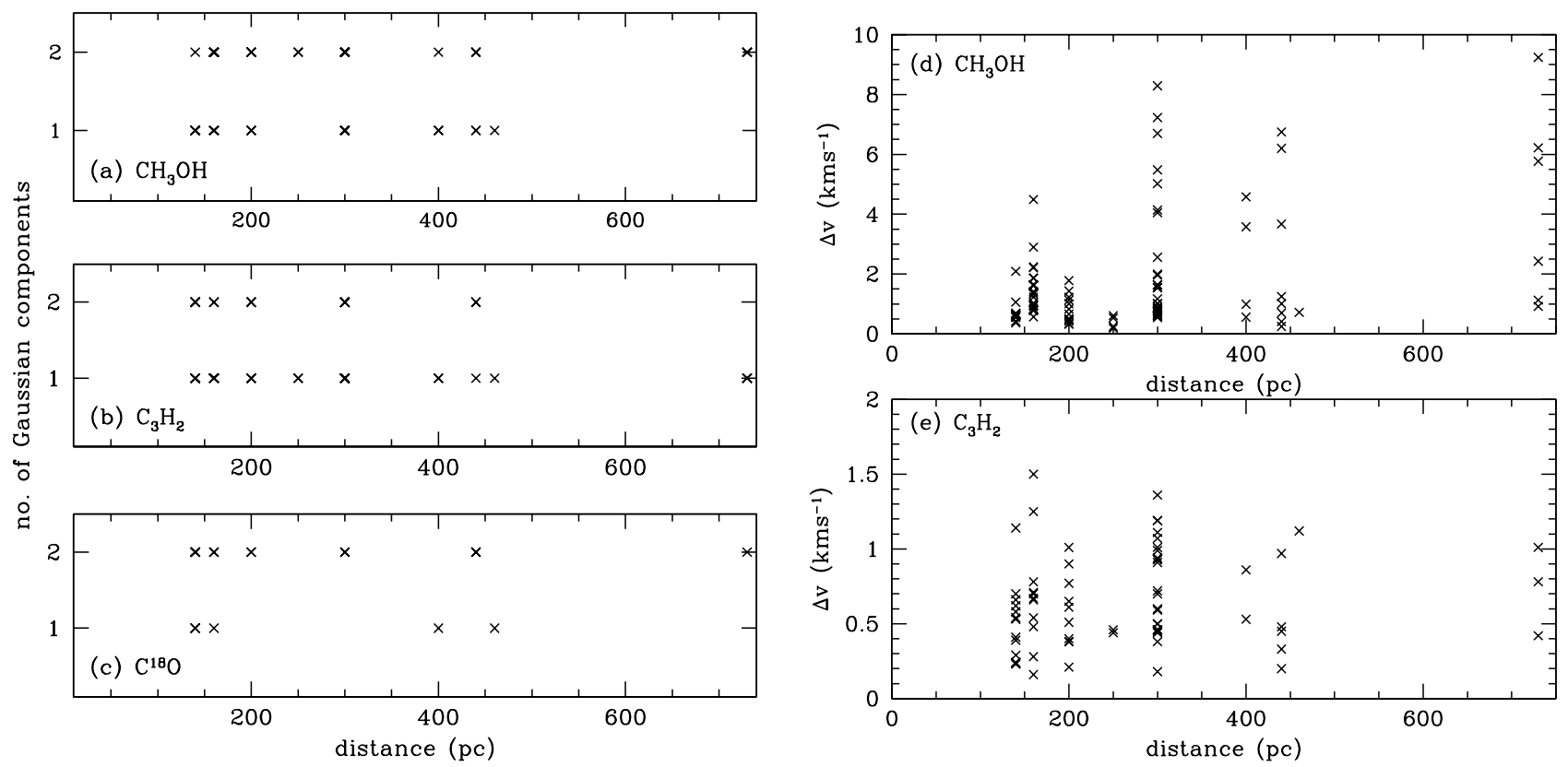

Fig. 3. a)-c) The number of detected components for each source as a function of distance to the source. d) $-\mathbf{e})$ The derived linewidths as a function of distance to the source.

Table 4. Weighted average of linewidths, by molecule and by class/position, with the error on the mean in parentheses. The number of positions detected in the component is given to the right in each column.

\begin{tabular}{|c|c|c|c|c|c|c|c|}
\hline Position & Molecule & $\left\langle\Delta v_{\text {broad }}\right\rangle \mathrm{km} \mathrm{s}^{-1}$ & $\#$ & $\left\langle\Delta v_{\text {narrow }}\right\rangle \mathrm{km} \mathrm{s}^{-1}$ & $\#$ & $\left\langle\Delta v_{\text {single }}\right\rangle \mathrm{km} \mathrm{s}^{-1}$ & $\#$ \\
\hline \multirow[t]{3}{*}{ All } & $\mathrm{CH}_{3} \mathrm{OH}$ & $1.46(.02)$ & 35 & $0.72(.01)$ & 35 & $0.69(.01)$ & 27 \\
\hline & $\mathrm{c}-\mathrm{C}_{3} \mathrm{H}_{2}$ & $0.59(.01)$ & 17 & $0.39(.01)$ & 17 & $0.59(.01)$ & 37 \\
\hline & $\mathrm{DCN}$ & .. & & . & & $0.64(.01)$ & 41 \\
\hline \multirow[t]{3}{*}{ Core 0} & $\mathrm{CH}_{3} \mathrm{OH}$ & $2.09(.03)$ & 13 & $0.95(.01)$ & 13 & $0.62(.01)$ & 8 \\
\hline & $\mathrm{c}-\mathrm{C}_{3} \mathrm{H}_{2}$ & $0.56(.01)$ & 6 & $0.30(.01)$ & 6 & $0.67(.01)$ & 14 \\
\hline & $\mathrm{DCN}$ & & & & & $0.65(.01)$ & 18 \\
\hline \multirow[t]{3}{*}{ Core I } & $\mathrm{CH}_{3} \mathrm{OH}$ & $0.99(.05)$ & 4 & $0.34(.03)$ & 4 & $0.89(.02)$ & 8 \\
\hline & $\mathrm{c}-\mathrm{C}_{3} \mathrm{H}_{2}$ & $0.69(.03)$ & 4 & $0.48(.01)$ & 4 & $0.55(.01)$ & 5 \\
\hline & $\mathrm{DCN}$ & .. & &. & & $0.54(.02)$ & 7 \\
\hline \multirow[t]{3}{*}{ Outflow 0} & $\mathrm{CH}_{3} \mathrm{OH}$ & $1.26(.02)$ & 17 & $0.61(.01)$ & 17 & $0.70(.01)$ & 7 \\
\hline & $\mathrm{c}-\mathrm{C}_{3} \mathrm{H}_{2}$ & $0.94(.03)$ & 6 & $0.40(.01)$ & 6 & $0.54(.01)$ & 16 \\
\hline & $\mathrm{DCN}$ & .. & & .. & & $0.64(.01)$ & 15 \\
\hline \multirow[t]{3}{*}{ Outflow I } & $\mathrm{CH}_{3} \mathrm{OH}$ & $0.69(.09)$ & 1 & $0.66(.16)$ & 1 & $0.60(.04)$ & 4 \\
\hline & $\mathrm{c}-\mathrm{C}_{3} \mathrm{H}_{2}$ & $0.33(.03)$ & 1 & $0.20(.05)$ & 1 & $0.72(.08)$ & 2 \\
\hline & $\mathrm{DCN}$ & .. & & .. & & $0.79(.13)$ & 1 \\
\hline
\end{tabular}

sources, the separation of the components can clearly be seen in the spectra (Fig. 1).

For $\mathrm{c}^{-} \mathrm{C}_{3} \mathrm{H}_{2}$ (Fig. 6b) the narrow component is also both red and blue-shifted with respect to the broad component, but there are more sources which have a narrow component which is blue-shifted with respect to the broad component. The differences in the central velocity between $\mathrm{c}_{-} \mathrm{C}_{3} \mathrm{H}_{2}$ narrow and broad components is generally $<0.7 \mathrm{~km} \mathrm{~s}^{-1}$. For the majority of sources, $v_{0}$ broad $\sim$ $v_{0}$ narrow for both $\mathrm{CH}_{3} \mathrm{OH}$ and $\mathrm{c}-\mathrm{C}_{3} \mathrm{H}_{2}$.

The single components of $\mathrm{CH}_{3} \mathrm{OH}$ are slightly redshifted relative to the same component in the $\mathrm{c}-\mathrm{C}_{3} \mathrm{H}_{2}$ lines, while the differences between the narrow and broad components of these two molecules are more evenly distributed. The single components of DCN are blue shifted with respect to the $\mathrm{CH}_{3} \mathrm{OH}$ single component lines towards the majority of sources.

The linewidths of emission we detect suggest that emission is arising in kinematically distinct regions. However, we only detect differences in central velocities of a few $x$ $0.1 \mathrm{~km} \mathrm{~s}^{-1}$ in the majority of sources.

\section{2. $\mathrm{CH}_{3} \mathrm{OH}$ rotation diagram analysis}

The emission from $\mathrm{CH}_{3} \mathrm{OH}$ has been analysed using the rotation diagram method, which can be applied to optically thin emission, where the molecules are in local thermodynamic equilibrium (LTE). This technique has 

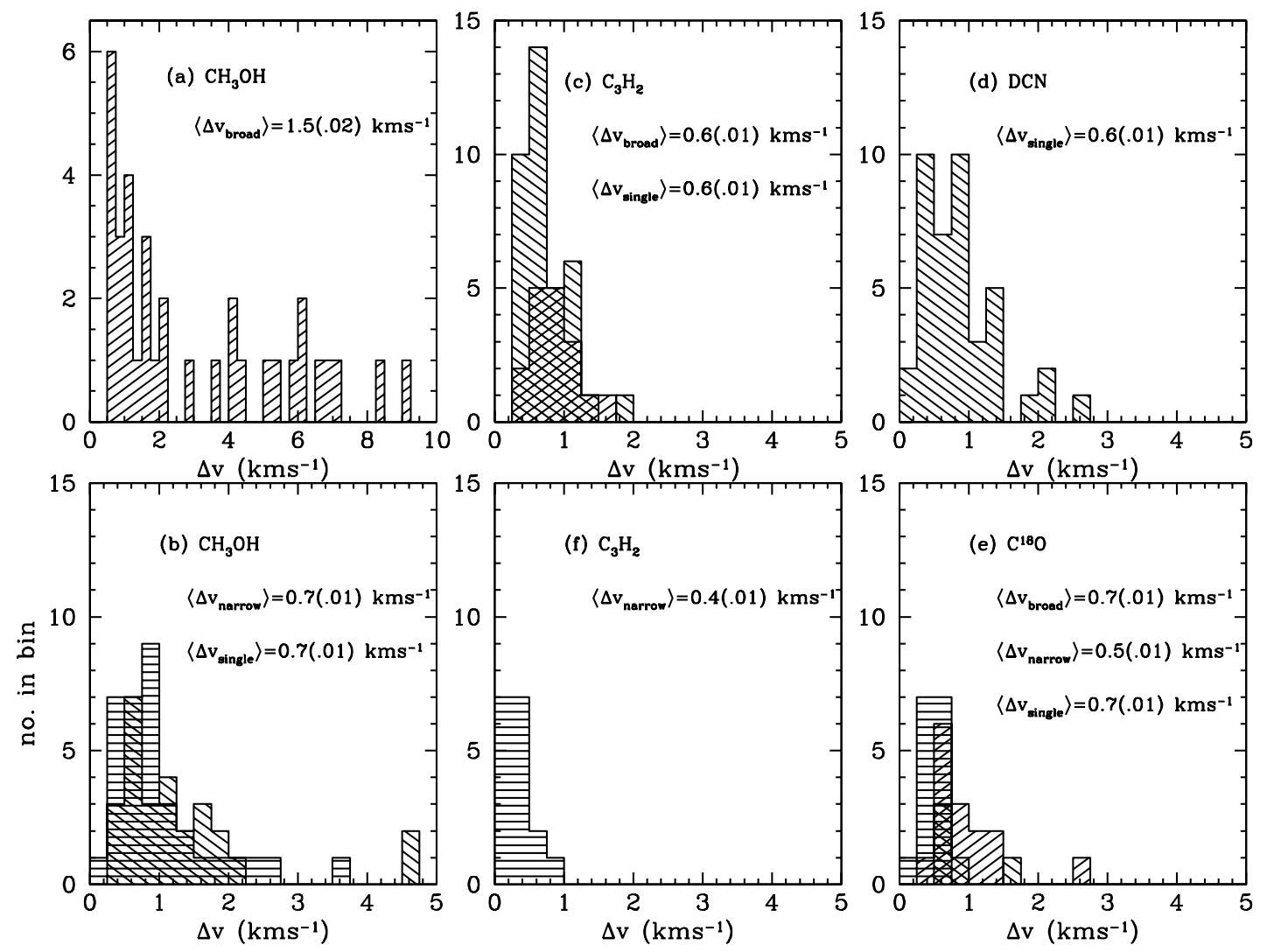

Fig. 4. Histograms of the linewidths of the single, broad and narrow components for each molecule detected towards all observed positions. Each plot is labelled with the molecule. The weighted average of the linewidth components for each molecule, with the error on the mean in parentheses, is shown on each plot. Left to right diagonally shaded histograms (/) are broad components, horizontally shaded histograms are narrow components, and right to left diagonally shaded $(\backslash)$ histograms are single components.
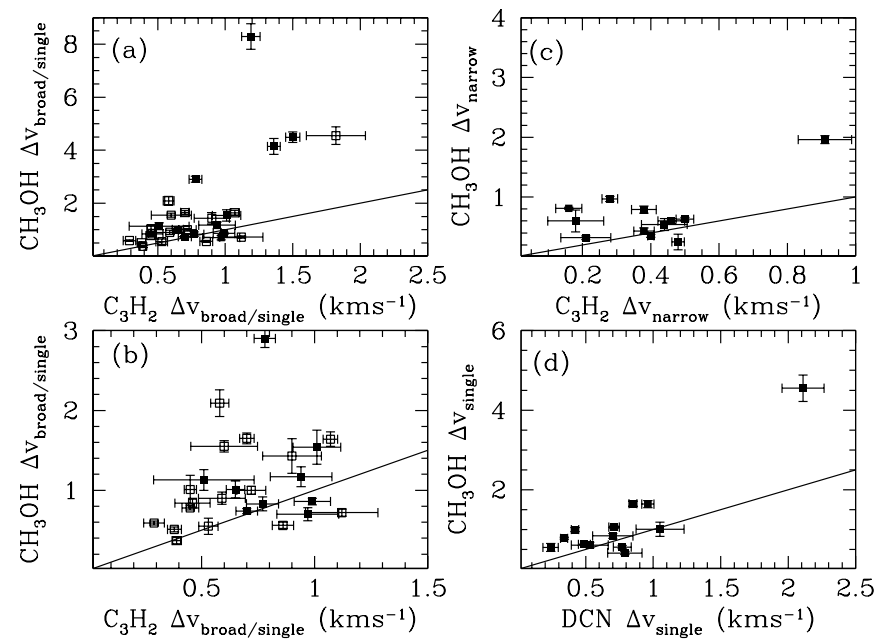

Fig. 5. A comparison of the relative linewidths of $\mathrm{CH}_{3} \mathrm{OH}$, c- $\mathrm{C}_{3} \mathrm{H}_{2}$ and DCN. b) shows the narrow linewidth section of a). In a) and b) filled squares denote the broad component.

been successfully used to analyse $\mathrm{CH}_{3} \mathrm{OH}$ emission in outflow regions (Helmich et al. 1994; Bachiller et al. 1998; Van Dishoeck et al. 1995). In star forming regions, previous observations of several transitions of $\mathrm{CH}_{3} \mathrm{OH}$ at different frequencies, observations of ${ }^{13} \mathrm{CH}_{3} \mathrm{OH}$ and statistical equilibrium calculations (Kalenskii et al. 1997) all suggest that the transitions are optically thin.

The rotation diagram method provides the rotational temperature, $T_{\text {rot }}$, and the total column density, $N_{\text {tot }}$, through the relation:

$\frac{3 k \int T_{\mathrm{mb}} \mathrm{d} v}{8 \pi^{3} \mu^{2} \nu S}=\frac{N_{\mathrm{u}}}{g_{\mathrm{u}}}=\frac{N_{\text {tot }}}{Q\left(T_{\text {rot }}\right)} \mathrm{e}^{-E_{\mathrm{u}}} / k T_{\text {rot }}$

where $T_{\mathrm{mb}}$ is calculated from the corrected source antenna temperature, $T_{R}^{*}$, using the corrected main beam efficiency, $T_{\mathrm{mb}}=T_{\mathrm{R}}^{*} / \eta_{\mathrm{m}}^{*} \cdot \int T_{\mathrm{mb}} \mathrm{d} v$ is the integrated line intensity, $\mu$ is the transition dipole moment, $S$ is the line strength, $N_{\mathrm{u}}$ is the level column density, $g_{\mathrm{u}}$ is the statistical weight, and $E_{\mathrm{u}}$ is the upper energy level. The partition function $Q\left(T_{\text {rot }}\right)=1.28 T_{\text {rot }}^{1.5}$ was adopted from Menten et al. (1986), and takes into account the equal populations of $A$ and $E$ species. The rotational constants and energies were obtained from the $J P L$ molecular line database (Pickett et al. 1998), and the values of $\mu^{2} S$ were taken from Anderson et al. (1990). Taking logarithms of the quantities in Eq. (2), the total column densities can then be calculated from a least-squares fitting of a straight line to the data. For methanol, the populations of the $E$ and $A$ species are usually assumed to be equal (Kalenskii et al. 1997), with the $A$ species having a slightly lower zero point energy. We have four $E$ type transitions, and 

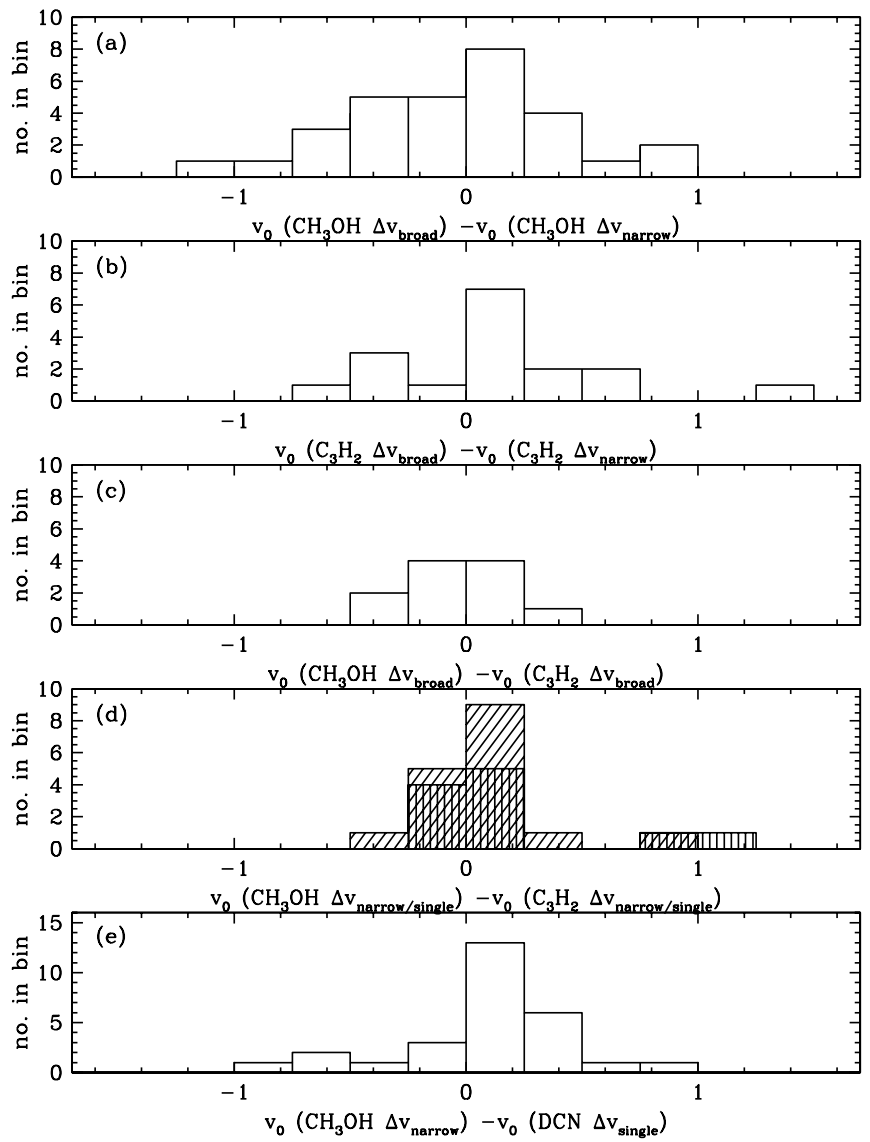

Fig. 6. Differences between the central velocities of $\mathrm{CH}_{3} \mathrm{OH}$, c- $\mathrm{C}_{3} \mathrm{H}_{2}$ and DCN components. For d) the difference between narrow components is shaded in vertical lines, while the difference between single components is shaded in diagonal lines.

one $A$ type transition in these observations, although not all of the spectra have detectable emission from the higher lying $E$ type transitions. The $A$ species transition is the strongest line in all of the sources, and has a good signal to noise ratio in most of the spectra. We have excluded the blended $E$ line from the rotation diagram fit, as we cannot derive the individual contributions to the emission line from the line fitting procedure. Although the methanol may be sub-thermally excited, the rotation diagram method will provide rotation temperatures that are at least lower limits on the kinetic temperature.

Rotation diagrams and the linear least squares fit are shown for four sources in Fig. 7, where all the lines except for the blended $E$ transitions have been included in the fit. The error bars are from uncertainties in the integrated line intensities, calculated from the uncertainties in the fit. S68N has two component lines, which are fitted separately, to give rotation temperatures of $11.9 \mathrm{~K}$ (broad) and $10.1 \mathrm{~K}$ (narrow), showing that the two components are associated with gas having similar rotation temperatures. For L1157, we derive rotation temperatures of $11.5 \mathrm{~K}$ (broad) and $14.7 \mathrm{~K}$ (narrow) towards the core position, and $8.8 \mathrm{~K}$ (broad) and $8.4 \mathrm{~K}$ (narrow) towards the outflow position. For L1527 and IRAM 04191, the lines show only a single component, giving rotation temperatures derived from the
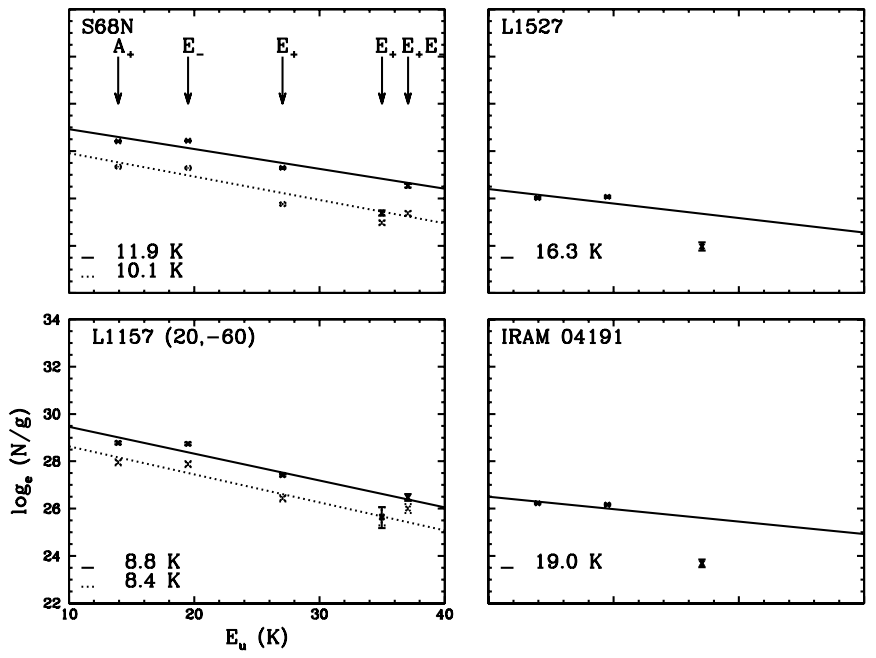

Fig. 7. Rotation diagrams for four of the sources in the sample. Arrows indicate the species giving rise to the transitions. The error bars are from the uncertainties in the integrated intensities. Rotation temperatures are produced from linear least square fits to the derived column densities. Squares and the solid line mark the broad component; crosses and a dotted line mark the narrow component. Top left: S68N, Class 0, two component fit. Top right: L1527, Class 0, single component fit. Bottom left: L1157 outflow position, Class 0, two component fit. Bottom right: IRAM 04191, Class 0, single component fit.

rotation diagram technique of $16.3 \mathrm{~K}$ (L1527) and $19.0 \mathrm{~K}$ (04191). The derived rotation temperatures for all the observed positions are given in Table 5 .

\subsection{Minimum values for rotation temperatures and column densities}

For those positions where only two $\mathrm{CH}_{3} \mathrm{OH}$ transitions were detected with reasonable signal to noise, or for analysing emission from $\mathrm{c}-\mathrm{C}_{3} \mathrm{H}_{2}$ and $\mathrm{DCN}$ where we only detected one transition, the rotation diagram method is unable to constrain the temperature and total column density. For these positions, Eq. (2) can be modified to determine a lower limit to the column density (Thompson et al. 1999). The rotation temperature can be approximated as $T_{\text {rot }}=\frac{2 E_{\mathrm{u}}}{3 k}$ for non-linear molecules, or as $T_{\text {rot }}=\frac{E_{\mathrm{u}}}{k}$ for linear molecules, based on the evaluation of the turning point of the temperature dependent part of Eq. (2):

$\frac{\mathrm{d}}{\mathrm{d} T_{\text {rot }}}\left(Q\left(T_{\text {rot }}\right) \mathrm{e}^{\frac{E_{u}}{k T_{\text {ex }}}}\right)=0$.

Since the second derivative shows that the rotation temperature approximations are turning points that are minima, they can be used to calculate a lower limit to the molecular column density, $N_{\min }$ :

$N_{\min }=\frac{3 k \int T_{\mathrm{mb}} \mathrm{d} v Q\left(\frac{2 E_{\mathrm{u}}}{3 k}\right) e^{3 / 2}}{8 \pi^{3} \mu^{2} \nu S} \quad$ non-linear 


$$
N_{\min }=\frac{3 k \int T_{\mathrm{mb}} \mathrm{d} v Q\left(\frac{E_{\mathrm{u}}}{k}\right) e}{8 \pi^{3} \mu^{2} \nu S} \quad \text { linear. }
$$

This analysis also assumes that the molecules are in LTE, and that the emission is optically thin. All molecular constants were obtained from the JPL molecular line database (Pickett et al. 1998), except for the $\mathrm{CH}_{3} \mathrm{OH}$ partition function and $\mu^{2} S$ values, which remain as described in Sect. 4.2. Table 5 displays the derived total column densities and rotation temperatures for the sources in Table 2. Where minimum values have been calculated, this has been indicated.

Due to the two hydrogen spins, $\mathrm{c}-\mathrm{C}_{3} \mathrm{H}_{2}$ possesses both ortho and para species, with an ortho-to-para ratio, based on statistical weights, of $3: 1$. Given the small energy difference between the ortho and para states $(\Delta E=2.35 \mathrm{~K}$, Turner 1998), the ratio should remain close to this even at the temperatures, $\sim 10 \mathrm{~K}$, of dark clouds. The detected transition is an ortho species, and so the calculated column density has been multiplied by $4 / 3$ before listing in Table 5 .

\section{4. $\mathrm{CH}_{3} \mathrm{OH}$ rotation temperatures}

Our observations suggest that emission from $\mathrm{CH}_{3} \mathrm{OH}$ may be sub-thermally excited towards low mass protostellar regions; we detect $\mathrm{CH}_{3} \mathrm{OH}$ emission from regions characterised by linewidths $<1 \mathrm{kms}^{-1}$, and $>5 \mathrm{kms}^{-1}$, but the derived rotation temperatures in both components are $\sim 10 \mathrm{~K}$. If the broader $\mathrm{CH}_{3} \mathrm{OH}$ component is arising in shock, or post-shock regions, characterised by higher kinetic temperatures, then we would expect to see higher rotation temperatures in the broader component material. Other observations of $\mathrm{CH}_{3} \mathrm{OH}$ towards star forming regions also suggest that the emission could be subthermally excited (Gibb \& Davis 1998; Bachiller et al. 1995).

In order to investigate the possibility of sub-thermal excitation, we have carried out an analysis using a statistical equilibrium model. Using this model, we can compare kinetic and rotation temperatures derived from the model with rotation temperatures obtained from the data, and so infer kinetic temperatures in the emission regions.

We have used a statistical equilibrium code developed by Walmsley et al. (1998), which has previously been used to analyse the excitation of $\mathrm{CH}_{3} \mathrm{OH}$ in star forming regions. The code considers $\mathrm{A}$ and $\mathrm{E}$ type transitions separately, and assumes that the two species have the same abundance. Levels up to $J=15$ and $K=3$ are included, with $\mathrm{CH}_{3} \mathrm{OH}$ energy levels based on Pickett et al. (1998) and collision rates from Haque et al. (1974). The parameter space for the model covers $\mathrm{CH}_{3} \mathrm{OH}$ column densities per linewidth of $10^{10}-10^{14} \mathrm{~cm}^{-2} / \mathrm{km} \mathrm{s}^{-1}, \mathrm{H}_{2}$ densities of $10^{3}-10^{7} \mathrm{~cm}^{-3}$ and kinetic temperatures of $5-100 \mathrm{~K}$. We have assumed equal gas and dust temperatures. In order to compare the model with our data, we have extracted the intensities for the 5 lines we detected, and produced rotation diagrams using the same method as for the data, described in Sect. 4.2 .

The results of the statistical equilibrium modelling are summarised in Fig. 8, which show the derived rotation temperatures and column densities as a function of kinetic temperature and $\mathrm{H}_{2}$ density. As Figs. $8 \mathrm{a}-\mathrm{f}$ show, the rotation temperature of these transitions is a poor tracer of the kinetic temperature. For kinetic temperatures of $100 \mathrm{~K}$, rotation temperatures vary from $3 \mathrm{~K}$ at $\mathrm{H}_{2}$ densities of $10^{4} \mathrm{~cm}^{-3}$, to $20 \mathrm{~K}$ at $\mathrm{H}_{2}$ densities of $10^{7} \mathrm{~cm}^{-3}$. For kinetic temperatures of $10 \mathrm{~K}$, rotation temperatures vary from $3 \mathrm{~K}$ at $\mathrm{H}_{2}$ densities of $10^{4} \mathrm{~cm}^{-3}$, to $9 \mathrm{~K}$ at $\mathrm{H}_{2}$ densities of $10^{7} \mathrm{~cm}^{-3}$. For number densities $\leq 10^{5} \mathrm{~cm}^{-3}$ the rotation temperature is essentially independent of the kinetic temperature. Even for a kinetic temperature of $100 \mathrm{~K}$, a number density $>10^{6} \mathrm{~cm}^{-3}$ is needed to obtain a rotation temperature of $\sim 10 \mathrm{~K}$, similar to that derived from the observations. This modelling suggests that despite the low rotation temperatures derived, the material traced by the $\mathrm{CH}_{3} \mathrm{OH}$ emission is both dense, $n\left(\mathrm{H}_{2}\right) \geq 10^{6} \mathrm{~cm}^{-3}$, and warm, with temperatures possibly as high as $100 \mathrm{~K}$.

From this statistical equilibrium analysis, it is clear that $\mathrm{CH}_{3} \mathrm{OH}$ emission is sub-thermally excited within star forming regions, and that kinetic temperatures could easily be warm enough to evaporate grain mantles. The rotation temperature will only give a lower limit to the kinetic temperature. However, the $\mathrm{CH}_{3} \mathrm{OH}$ column densities that we derive from our data are more accurately constrained by the rotation diagram method. This is in agreement with similar work carried out using statistical equilibrium codes (Bachiller et al. 1995; Johnston et al. 1992) and radiative transfer modelling using large velocity gradient codes (Gibb \& Davis 1998; Bachiller et al. 1998).

\subsection{Column densities}

We find $\mathrm{CH}_{3} \mathrm{OH}$ column densities of a few $\times 10^{13}$ to a few $\times 10^{14} \mathrm{~cm}^{-2}$, with the larger values usually in the broad component. The column densities derived for $\mathrm{A}$ type $\mathrm{CH}_{3} \mathrm{OH}$ using the minimum column density calculations are within a factor of a few of those derived for both species using the rotation diagram method. For $\mathrm{c}-\mathrm{C}_{3} \mathrm{H}_{2}$, we derive minimum column densities of a few $\times 10^{12}$ to a few $\times 10^{13} \mathrm{~cm}^{-2}$, and a few $\times 10^{11}$ to a few $\times 10^{12} \mathrm{~cm}^{-2}$ for DCN. The derived column densities may be lower limits, since we have assumed that the emission fills the beam.

Averaged over all of the sources the broad component has about twice the column density of the narrow component. Figure $9 \mathrm{a}-\mathrm{b}$ shows a histogram of the ratio of the total column densities from the two regions $\left(\frac{N \text { (broad })}{N \text { (narrow })}\right)$. As can be seen, the broad component of the $\mathrm{CH}_{3} \mathrm{OH}$ and the $\mathrm{c}_{-} \mathrm{C}_{3} \mathrm{H}_{2}$ lines are tracing higher column density regions than the narrow component. Although the $\mathrm{CH}_{3} \mathrm{OH}$ column densities differ by only a small factor, we need estimates of $\mathrm{H}_{2}$ column densities in the two regions to determine whether $\mathrm{CH}_{3} \mathrm{OH}$ abundances show greater enhancements in the broad material. 
Table 5. Temperatures and column densities calculated for $\mathrm{CH}_{3} \mathrm{OH}$ from linear least square fits to the rotation diagram. Uncertainties (1 sigma) are given in parentheses. For sources with two components the narrow component values are shown first. For some sources, and for $\mathrm{c}^{-} \mathrm{C}_{3} \mathrm{H}_{2}$ and DCN, we were unable to constrain the temperature and total column densities from the rotation diagram fit, and so approximations to the rotation temperature and minimum values for the total column density have been included, as described in the text. Offset positions are given in arcsec.

\begin{tabular}{|c|c|c|c|c|c|c|c|}
\hline Source & Molecule & $\begin{array}{r}\text { Width } \\
\mathrm{km} \mathrm{s}^{-1}\end{array}$ & $\begin{array}{r}T_{\text {rot }} \\
\mathrm{K}\end{array}$ & $\begin{array}{r}N(\mathrm{~mol}) \\
\times 10^{13} \mathrm{~cm}^{-3}\end{array}$ & $\begin{array}{r}\text { Width } \\
\mathrm{km} \mathrm{s}^{-1}\end{array}$ & $\begin{array}{r}T_{\text {rot }} \\
\mathrm{K}\end{array}$ & $\begin{array}{r}N(\mathrm{~mol}) \\
\times 10^{13} \mathrm{~cm}^{-3}\end{array}$ \\
\hline \multirow[t]{3}{*}{ L1448N } & $\mathrm{CH}_{3} \mathrm{OH}$ & 0.86 & $9.32(0.64)$ & $8.68(1.19)$ & 0.60 & $11.85(1.46)$ & $4.10(0.83)$ \\
\hline & $\mathrm{C}_{3} \mathrm{H}_{2}$ & 0.99 & $\sim 10.70$ & $\geq 0.88$ & 0.46 & $\sim 10.70$ & $\geq 0.28$ \\
\hline & DCN & 0.76 & $\sim 10.43$ & $\geq 0.23$ & .. & .. & .. \\
\hline \multirow[t]{3}{*}{ L1448NW } & $\mathrm{CH}_{3} \mathrm{OH}$ & 0.74 & $9.74(0.77)$ & $6.39(0.97)$ & 0.63 & $10.31(1.07)$ & $5.68(1.01)$ \\
\hline & $\mathrm{C}_{3} \mathrm{H}_{2}$ & 0.70 & $\sim 10.70$ & $\geq 0.54$ & 0.50 & $\sim 10.70$ & $\geq 0.45$ \\
\hline & DCN & 0.83 & $\sim 10.43$ & $\geq 0.29$ & & .. & .. \\
\hline \multirow[t]{3}{*}{$\mathrm{L} 1448 \mathrm{~N}(+25,-35)$} & $\mathrm{CH}_{3} \mathrm{OH}$ & 4.14 & $13.91(2.61)$ & $13.76(3.48)$ & 0.79 & $14.65(4.48)$ & $6.87(2.40)$ \\
\hline & $\mathrm{C}_{3} \mathrm{H}_{2}$ & 1.36 & $\sim 10.70$ & $\geq 1.31$ & 0.38 & $\sim 10.70$ & $\geq 0.22$ \\
\hline & $\mathrm{DCN}$ & 1.17 & $\sim 10.43$ & $\geq 0.32$ & .. & .. & .. \\
\hline \multirow[t]{3}{*}{$\mathrm{L} 1448 \mathrm{~N}(+57,-110)$} & $\mathrm{CH}_{3} \mathrm{OH}$ & 1.54 & $11.22(4.09)$ & $4.51(2.68)$ & 0.54 & $\sim 9.29$ & $\geq 1.88$ \\
\hline & $\mathrm{C}_{3} \mathrm{H}_{2}$ & 1.01 & $\sim 10.70$ & $\geq 0.49$ & 0.44 & $\sim 10.70$ & $\geq 0.24$ \\
\hline & $\mathrm{DCN}$ & 1.20 & $\sim 10.43$ & $\geq 0.17$ & .. & .. & .. \\
\hline \multirow[t]{3}{*}{ L1448C } & $\mathrm{CH}_{3} \mathrm{OH}$ & 1.64 & $11.35(1.34)$ & $8.50(1.61)$ & .. & .. & .. \\
\hline & $\mathrm{C}_{3} \mathrm{H}_{2}$ & 1.07 & $\sim 10.70$ & $\geq 1.12$ & .. & .. & .. \\
\hline & DCN & 0.96 & $\sim 10.43$ & $\geq 0.45$ & .. & .. & .. \\
\hline \multirow[t]{3}{*}{ L1448R3 } & $\mathrm{CH}_{3} \mathrm{OH}$ & 7.23 & $20.40(4.62)$ & $14.59 \overline{(3.09)}$ & 1.02 & $13.38(4.47)$ & $2.98(1.30)$ \\
\hline & $\mathrm{C}_{3} \mathrm{H}_{2}$ & 1.19 & $\sim 10.70$ & $\geq 0.50$ & .. & .. & .. \\
\hline & $\mathrm{DCN}$ & 1.30 & $\sim 10.43$ & $\geq 0.09$ & .. & .. & .. \\
\hline \multirow[t]{3}{*}{ I03282 } & $\mathrm{CH}_{3} \mathrm{OH}$ & 1.00 & $\sim 9.29$ & $\geq 3.45$ & .. & .. & .. \\
\hline & $\mathrm{C}_{3} \mathrm{H}_{2}$ & 0.72 & $\sim 10.70$ & $\geq 0.24$ & .. & .. & .. \\
\hline & $\mathrm{DCN}$ & 0.42 & $\sim 10.43$ & $\geq 0.07$ & .. & .. & .. \\
\hline \multirow[t]{3}{*}{ НH211 } & $\mathrm{CH}_{3} \mathrm{OH}$ & 4.05 & $8.91(3.78)$ & $2.81 \overline{(2.28)}$ & 0.69 & $10.70(0.75)$ & $6.33(0.74)$ \\
\hline & $\mathrm{C}_{3} \mathrm{H}_{2}$ & 0.50 & $\sim 10.70$ & $\geq 0.28$ & .. & .. & .. \\
\hline & $\mathrm{DCN}$ & 0.48 & $\sim 10.43$ & $\geq 0.07$ & .. & .. & .. \\
\hline \multirow[t]{3}{*}{$\mathrm{HH} 211(-14,7)$} & $\mathrm{CH}_{3} \mathrm{OH}$ & 0.84 & $\sim 9.29$ & $\geq 2.83$ & .. & .. & .. \\
\hline & $\mathrm{C}_{3} \mathrm{H}_{2}$ & 0.46 & $\sim 10.70$ & $\geq 0.09$ & .. & .. & .. \\
\hline & DCN & 0.70 & $\sim 10.43$ & $\geq 0.06$ & .. & .. & .. \\
\hline \multirow[t]{3}{*}{ HH211(18, -9) } & $\mathrm{CH}_{3} \mathrm{OH}$ & 0.78 & $16.18(2.24)$ & $6.24(0.92)$ & .. & .. & .. \\
\hline & $\mathrm{C}_{3} \mathrm{H}_{2}$ & 0.45 & $\sim 10.70$ & $\geq 0.24$ & .. & .. & .. \\
\hline & $\mathrm{DCN}$ & 0.34 & $\sim 10.43$ & $\geq 0.03$ & .. & .. & .. \\
\hline \multirow[t]{2}{*}{ HH211(41, 14) } & $\mathrm{CH}_{3} \mathrm{OH}$ & 1.17 & $\sim 9.29$ & $\geq 3.24$ & 0.60 & $\sim 9.29$ & $\geq 1.15$ \\
\hline & $\mathrm{C}_{3} \mathrm{H}_{2}$ & 0.94 & $\sim 10.70$ & $\geq 0.19$ & 0.18 & $\sim 10.70$ & $\geq 0.06$ \\
\hline \multirow[t]{2}{*}{ HH211 $(41,-14)$} & $\mathrm{CH}_{3} \mathrm{OH}$ & 1.55 & $12.30(1.71)$ & $5.60(\overline{1} .09)$ & .. & .. & $\begin{array}{l}- \\
-\end{array}$ \\
\hline & $\mathrm{C}_{3} \mathrm{H}_{2}$ & 0.60 & $\sim 10.70$ & $\geq 0.07$ & .. & .. & .. \\
\hline \multirow[t]{2}{*}{ HH211 $(-43,14)$} & $\mathrm{CH}_{3} \mathrm{OH}$ & 0.90 & $15.92(3.10)$ & $3.11(0.73)$ & .. & .. & .. \\
\hline & $\mathrm{C}_{3} \mathrm{H}_{2}$ & 0.59 & $\sim 10.70$ & $\geq 0.07$ & .. & .. & .. \\
\hline \multirow[t]{3}{*}{ IRAM 04191} & $\mathrm{CH}_{3} \mathrm{OH}$ & 0.63 & $19.05(2.50)$ & $5.85(0.68)$ & .. & .. & .. \\
\hline & $\mathrm{C}_{3} \mathrm{H}_{2}$ & 0.41 & $\sim 10.70$ & $\geq 0.28$ & 0.24 & $\sim 10.70$ & $\geq 0.04$ \\
\hline & $\mathrm{DCN}$ & 0.49 & $\sim 10.43$ & $\geq 0.03$ & .. & .. & .. \\
\hline \multirow[t]{2}{*}{ IRAM 04191 $(6,17)$} & $\mathrm{CH}_{3} \mathrm{OH}$ & 0.59 & $11.18(1.03)$ & $4.87(0.72)$ & .. & .. & .. \\
\hline & $\mathrm{C}_{3} \mathrm{H}_{2}$ & 0.29 & $\sim 10.70$ & $\geq 0.09$ & .. & .. & .. \\
\hline \multirow[t]{2}{*}{ Haro6-10 } & $\mathrm{CH}_{3} \mathrm{OH}$ & 0.37 & $\sim 9.29$ & $\geq 1.23$ & .. & .. & .. \\
\hline & $\mathrm{C}_{3} \mathrm{H}_{2}$ & 0.39 & $\sim 10.70$ & $\geq 0.26$ & .. & .. & .. \\
\hline L1551IRS5 & $\mathrm{CH}_{3} \mathrm{OH}$ & 1.06 & $12.02(0.73)$ & $7.74(0.67)$ & .. & .. & .. \\
\hline & $\mathrm{C}_{3} \mathrm{H}_{2}$ & 1.14 & $\sim 10.70$ & $\geq 0.17$ & 0.66 & $\sim 10.70$ & $\geq 0.54$ \\
\hline & $\mathrm{DCN}$ & 0.71 & $\sim 10.43$ & $\geq 0.18$ & .. & .. & .. \\
\hline L1551NE & $\mathrm{CH}_{3} \mathrm{OH}$ & 2.09 & $15.07(4.69)$ & $5.18(1.83)$ & .. & .. & .. \\
\hline & $\mathrm{C}_{3} \mathrm{H}_{2}$ & 0.58 & $\sim 10.70$ & $\geq 0.28$ & .. & .. & .. \\
\hline $\operatorname{L} 1551 \mathrm{NE}(-168,-96)$ & $\mathrm{CH}_{3} \mathrm{OH}$ & 0.69 & $9.04(2.06)$ & $2.82(1.29)$ & 0.66 & $9.46(4.06)$ & $1.42(1.21)$ \\
\hline & $\mathrm{C}_{3} \mathrm{H}_{2}$ & 0.62 & $\sim 10.70$ & $\geq 0.19$ & .. & .. & .. \\
\hline L1535 & $\mathrm{CH}_{3} \mathrm{OH}$ & 0.61 & $\sim 9.29$ & $\geq 5.65$ & .. & .. & .. \\
\hline & $\mathrm{C}_{3} \mathrm{H}_{2}$ & 0.70 & $\sim 10.70$ & $\geq 0.35$ & 0.23 & $\sim 10.70$ & $\geq 0.15$ \\
\hline & $\mathrm{DCN}$ & 0.53 & $\sim 10.43$ & $\geq 0.05$ & .. & .. & .. \\
\hline TMR1 & $\mathrm{CH}_{3} \mathrm{OH}$ & 0.55 & $\sim 9.29$ & $\geq 0.84$ & .. & .. & .. \\
\hline & $\mathrm{C}_{3} \mathrm{H}_{2}$ & 0.53 & $\sim 10.70$ & $\geq 0.21$ & .. & .. & .. \\
\hline & $\mathrm{DCN}$ & 0.24 & $\sim 10.43$ & $\geq 0.03$ & .. & .. & .. \\
\hline L1527 & $\mathrm{CH}_{3} \mathrm{OH}$ & 0.42 & $16.34(2.67)$ & $4.57 \overline{(0.80)}$ & .. & .. & .. \\
\hline & $\mathrm{C}_{3} \mathrm{H}_{2}$ & 0.54 & $\sim 10.70$ & $\geq 0.71$ & 0.24 & $\sim 10.70$ & $\geq 0.13$ \\
\hline
\end{tabular}


Table 5. continued.

\begin{tabular}{|c|c|c|c|c|c|c|c|}
\hline Source & Molecule & $\begin{array}{r}\text { Width } \\
\mathrm{km} \mathrm{s}^{-1}\end{array}$ & $\begin{array}{r}T_{\text {rot }} \\
\mathrm{K}\end{array}$ & $\begin{array}{r}N(\mathrm{~mol}) \\
\times 10^{13} \mathrm{~cm}^{-3}\end{array}$ & $\begin{array}{r}\text { Width } \\
\mathrm{km} \mathrm{s}^{-1}\end{array}$ & $\begin{array}{r}T_{\text {rot }} \\
\mathrm{K}\end{array}$ & $\begin{array}{r}N(\mathrm{~mol}) \\
\times 10^{13} \mathrm{~cm}^{-3}\end{array}$ \\
\hline \multirow[t]{3}{*}{ RNO43 } & $\mathrm{CH}_{3} \mathrm{OH}$ & 0.56 & $10.61(1.75)$ & $1.65(0.45)$ & .. & .. & .. \\
\hline & $\mathrm{C}_{3} \mathrm{H}_{2}$ & 0.86 & $\sim 10.70$ & $\geq 0.24$ & .. & .. & .. \\
\hline & DCN & 0.77 & $\sim 10.43$ & $\geq 0.09$ & .. & .. & .. \\
\hline \multirow[t]{3}{*}{ HH25 } & $\mathrm{CH}_{3} \mathrm{OH}$ & 3.58 & 20.61(7.05) & $8.96(2.55)$ & 0.99 & $11.26(1.44)$ & $4.24(0.84)$ \\
\hline & $\mathrm{C}_{3} \mathrm{H}_{2}$ & 0.53 & $\sim 10.70$ & $\geq 0.15$ & .. & .. & .. \\
\hline & DCN & 0.85 & $\sim 10.43$ & $\geq 0.04$ & .. & .. & .. \\
\hline HH25 $(224,30)$ & $\mathrm{CH}_{3} \mathrm{OH}$ & 4.58 & $13.33(2.41)$ & $3.73(0.97)$ & .. & .. & .. \\
\hline \multirow[t]{2}{*}{ HH111 } & $\mathrm{CH}_{3} \mathrm{OH}$ & 0.72 & $10.80(1.67)$ & $3.77(0.92)$ & .. & .. & .. \\
\hline & $\mathrm{C}_{3} \mathrm{H}_{2}$ & 1.12 & $\sim 10.70$ & $\geq 0.21$ & .. & .. & .. \\
\hline \multirow[t]{3}{*}{ VLA1623 } & $\mathrm{CH}_{3} \mathrm{OH}$ & 1.65 & $10.33(0.78)$ & $8.30(1.10)$ & .. & .. & .. \\
\hline & $\mathrm{C}_{3} \mathrm{H}_{2}$ & 0.70 & $\sim 10.70$ & $\geq 0.47$ & .. & .. & .. \\
\hline & $\mathrm{DCN}$ & 0.85 & $\sim 10.43$ & $\geq 0.36$ & .. & .. & .. \\
\hline \multirow[t]{3}{*}{ VLA1623(-42, 16) } & $\mathrm{CH}_{3} \mathrm{OH}$ & 1.86 & $10.74(0.91)$ & $10.96(1.52)$ & 0.79 & $24.23(7.58)$ & $2.17(0.68)$ \\
\hline & $\mathrm{C}_{3} \mathrm{H}_{2}$ & 1.25 & $\sim 10.70$ & $\geq 0.13$ & .. & .. & .. \\
\hline & $\mathrm{DCN}$ & 1.49 & $\sim 10.43$ & $\geq 0.10$ & .. & .. & .. \\
\hline \multirow[t]{3}{*}{$\operatorname{VLA} 1623(45,-24)$} & $\mathrm{CH}_{3} \mathrm{OH}$ & 1.41 & $\sim 9.29$ & $\geq 5.02$ & 0.84 & $\sim 9.29$ & $\geq 1.88$ \\
\hline & $\mathrm{C}_{3} \mathrm{H}_{2}$ & 0.66 & $\sim 10.70$ & $\geq 0.28$ & .. & .. & .. \\
\hline & $\mathrm{DCN}$ & 0.82 & $\sim 10.43$ & $\geq 0.25$ & .. & .. & .. \\
\hline \multirow[t]{3}{*}{ VLA1623(16, 76) } & $\mathrm{CH}_{3} \mathrm{OH}$ & 1.61 & $7.76(0.57)$ & $16.38(2.97)$ & 0.92 & $\sim 9.29$ & $\geq 2.62$ \\
\hline & $\mathrm{C}_{3} \mathrm{H}_{2}$ & 0.71 & $\sim 10.70$ & $\geq 0.50$ & .. & .. & .. \\
\hline & $\mathrm{DCN}$ & 1.30 & $\sim 10.43$ & $\geq 0.28$ & .. & .. & .. \\
\hline \multirow[t]{3}{*}{ VLA1623(16, 31) } & $\mathrm{CH}_{3} \mathrm{OH}$ & 1.64 & $11.13(1.59)$ & $7.16(1.74)$ & 0.57 & $12.73(3.53)$ & $2.91(1.17)$ \\
\hline & $\mathrm{C}_{3} \mathrm{H}_{2}$ & 0.67 & $\sim 10.70$ & $\geq 0.65$ & .. & .. & ... \\
\hline & $\mathrm{DCN}$ & 0.67 & $\sim 10.43$ & $\geq 0.31$ & .. & .. & .. \\
\hline \multirow[t]{3}{*}{$\operatorname{VLA} 1623(45,6)$} & $\mathrm{CH}_{3} \mathrm{OH}$ & 0.96 & $\sim 9.29$ & $\geq 2.20$ & 0.78 & $\sim 9.29$ & $\geq 4.71$ \\
\hline & $\mathrm{C}_{3} \mathrm{H}_{2}$ & 0.54 & $\sim 10.70$ & $\geq 0.41$ & .. & .. & .. \\
\hline & $\mathrm{DCN}$ & 0.53 & $\sim 10.43$ & $\geq 0.36$ & .. & .. & .. \\
\hline \multirow[t]{2}{*}{ VLA1623(-71, 36) } & $\mathrm{CH}_{3} \mathrm{OH}$ & 2.24 & $8.74(1.76)$ & $10.64 \overline{(5.30)}$ & 1.03 & $8.34(3.49)$ & $4.03(3.72)$ \\
\hline & $\mathrm{C}_{3} \mathrm{H}_{2}$ & 0.48 & $\sim 10.70$ & $\geq 0.19$ & .. & , & .. \\
\hline LFAM1 & $\mathrm{CH}_{3} \mathrm{OH}$ & 2.21 & $8.63(1.88)$ & $7.68 \overline{(4.13)}$ & 1.36 & $10.42(4.88)$ & $6.11(5.23)$ \\
\hline EL29 & $\mathrm{CH}_{3} \mathrm{OH}$ & 1.32 & $\sim 9.29$ & $\geq 0.73$ & .. & .. & .. \\
\hline $\mathrm{I} 16244$ & $\mathrm{CH}_{3} \mathrm{OH}$ & 1.23 & $11.82(2.02)$ & $2.87(0.80)$ & .. & .. & .. \\
\hline $\mathrm{I} 16244(35,14)$ & $\mathrm{CH}_{3} \mathrm{OH}$ & 1.87 & $13.93(3.79)$ & $2.52(0.95)$ & .. & .. & .. \\
\hline \multirow[t]{3}{*}{$\mathrm{I} 16293$} & $\mathrm{CH}_{3} \mathrm{OH}$ & 4.49 & $16.22(1.10)$ & $16.65(1.61)$ & 0.97 & $9.52(0.73)$ & $7.85(1.14)$ \\
\hline & $\mathrm{C}_{3} \mathrm{H}_{2}$ & 1.50 & $\sim 10.70$ & $\geq 0.77$ & 0.28 & $\sim 10.70$ & $\geq 0.13$ \\
\hline & $\mathrm{DCN}$ & 1.90 & $\sim 10.43$ & $\geq 0.44$ & .. & .. & .. \\
\hline \multirow[t]{3}{*}{$\mathrm{I} 16293(60,-45)$} & $\mathrm{CH}_{3} \mathrm{OH}$ & 2.90 & $11.27(0.58)$ & $14.59(1.35)$ & 0.81 & $9.38(0.38)$ & $11.31(0.88)$ \\
\hline & $\mathrm{C}_{3} \mathrm{H}_{2}$ & 0.78 & $\sim 10.70$ & $\geq 0.58$ & 0.16 & $\sim 10.70$ & $\geq 0.06$ \\
\hline & $\mathrm{DCN}$ & 0.73 & $\sim 10.43$ & $\geq 0.30$ & .. & .. & . \\
\hline \multirow[t]{3}{*}{ L483 } & $\mathrm{CH}_{3} \mathrm{OH}$ & 0.83 & $\sim 9.29$ & $\geq 1.99$ & 0.43 & $8.34(1.34)$ & $2.59(0.99)$ \\
\hline & $\mathrm{C}_{3} \mathrm{H}_{2}$ & 0.77 & $\sim 10.70$ & $\geq 0.21$ & 0.38 & $\sim 10.70$ & $\geq 0.30$ \\
\hline & $\mathrm{DCN}$ & 0.29 & $\sim 10.43$ & $\geq 0.04$ & .. & .. & ... \\
\hline \multirow[t]{3}{*}{$\mathrm{L} 483(-30,5)$} & $\mathrm{CH}_{3} \mathrm{OH}$ & 1.13 & $23.75(5.65)$ & $4.19 \overline{(1.05)}$ & 0.35 & $\sim 9.29$ & $\geq 2.72$ \\
\hline & $\mathrm{C}_{3} \mathrm{H}_{2}$ & 0.51 & $\sim 10.70$ & $\geq 0.07$ & 0.40 & $\sim 10.70$ & $\geq 0.65$ \\
\hline & $\mathrm{DCN}$ & 0.44 & $\sim 10.43$ & $\geq 0.09$ & .. & .. & .. \\
\hline $\mathrm{L} 483(40,-5)$ & $\mathrm{CH}_{3} \mathrm{OH}$ & 0.66 & $\sim 9.29$ & $\geq 2.20$ & 0.48 & $\sim 9.29$ & $\geq 0.42$ \\
\hline & $\mathrm{C}_{3} \mathrm{H}_{2}$ & 0.61 & $\sim 10.70$ & $\geq 0.28$ & .. & .. & .. \\
\hline & $\mathrm{DCN}$ & 0.67 & $\sim 10.43$ & $\geq 0.05$ & .. & .. & .. \\
\hline L483SW & $\mathrm{CH}_{3} \mathrm{OH}$ & 0.51 & $\sim 9.29$ & $\geq 2.09$ & .. & .. & .. \\
\hline & $\mathrm{C}_{3} \mathrm{H}_{2}$ & 0.38 & $\sim 10.70$ & $\geq 0.15$ & .. & .. & .. \\
\hline $\mathrm{S} 68 \mathrm{~N}$ & $\mathrm{CH}_{3} \mathrm{OH}$ & 6.70 & $11.87(0.30)$ & $44.75(1.81)$ & 1.63 & $10.09(0.32)$ & $14.75(0.87)$ \\
\hline & $\mathrm{C}_{3} \mathrm{H}_{2}$ & 1.11 & $\sim 10.70$ & $\geq 0.52$ & .. & .. & .. \\
\hline & $\mathrm{DCN}$ & 1.41 & $\sim 10.43$ & $\geq 0.15$ & .. & .. & .. \\
\hline SMM1 & $\mathrm{CH}_{3} \mathrm{OH}$ & 5.48 & $8.31(0.42)$ & $24.86 \overline{(3.29)}$ & 2.56 & $8.13(0.40)$ & $18.61(2.54)$ \\
\hline & $\mathrm{C}_{3} \mathrm{H}_{2}$ & 0.93 & $\sim 10.70$ & $\geq 0.17$ & .. & .. & .. \\
\hline & DCN & 0.43 & $\sim 10.43$ & $\geq 0.05$ & .. & .. & .. \\
\hline SMM4 & $\mathrm{CH}_{3} \mathrm{OH}$ & 8.29 & $9.39(0.54)$ & $20.01(2.72)$ & 1.96 & $12.14(1.10)$ & $16.46(2.14)$ \\
\hline & $\mathrm{C}_{3} \mathrm{H}_{2}$ & 1.19 & $\sim 10.70$ & $\geq 0.63$ & 0.91 & $\sim 10.70$ & $\geq 0.28$ \\
\hline & $\mathrm{DCN}$ & 2.04 & $\sim 10.43$ & $\geq 0.14$ & .. & .. & .. \\
\hline Serpens & $\mathrm{CH}_{3} \mathrm{OH}$ & 5.02 & $\sim 9.29$ & $\geq 5.75$ & 2.00 & $9.34(1.12)$ & $7.55(1.85)$ \\
\hline & $\mathrm{C}_{3} \mathrm{H}_{2}$ & 0.93 & $\sim 10.70$ & $\geq 0.28$ & .. & ... & .. \\
\hline
\end{tabular}


Table 5. continued.

\begin{tabular}{|c|c|c|c|c|c|c|c|}
\hline Source & Molecule & $\begin{array}{r}\text { Width } \\
\mathrm{km} \mathrm{s}^{-1}\end{array}$ & $\begin{array}{r}T_{\text {rot }} \\
\mathrm{K}\end{array}$ & $\begin{array}{r}N(\mathrm{~mol}) \\
\times 10^{13} \mathrm{~cm}^{-3}\end{array}$ & $\begin{array}{r}\text { Width } \\
\mathrm{km} \mathrm{s}^{-1}\end{array}$ & $\begin{array}{r}T_{\text {rot }} \\
\mathrm{K}\end{array}$ & $\begin{array}{r}N(\mathrm{~mol}) \\
\times 10^{13} \mathrm{~cm}^{-3}\end{array}$ \\
\hline \multirow[t]{3}{*}{ B335 } & $\mathrm{CH}_{3} \mathrm{OH}$ & 0.61 & $\sim 9.29$ & $\geq 1.25$ & 0.25 & $\sim 9.29$ & $\geq 0.31$ \\
\hline & $\mathrm{C}_{3} \mathrm{H}_{2}$ & 0.44 & $\sim 10.70$ & $\geq 0.17$ & .. & .. & .. \\
\hline & $\mathrm{DCN}$ & 0.39 & $\sim 10.43$ & $\geq 0.01$ & .. & .. & .. \\
\hline \multirow[t]{3}{*}{ B335 $(-60,24)$} & $\mathrm{CH}_{3} \mathrm{OH}$ & 0.53 & $\sim 9.29$ & $\geq 1.25$ & 0.19 & $\sim 9.29$ & $\geq 0.31$ \\
\hline & $\mathrm{C}_{3} \mathrm{H}_{2}$ & 0.46 & $\sim 10.70$ & $\geq 0.19$ & .. & .. & .. \\
\hline & $\mathrm{DCN}$ & 0.19 & $\sim 10.43$ & $\geq 0.01$ & .. & .. & .. \\
\hline \multirow[t]{3}{*}{ I 20126} & $\mathrm{CH}_{3} \mathrm{OH}$ & 4.55 & $14.80(2.28)$ & $9.07(1.84)$ & .. & .. & .. \\
\hline & $\mathrm{C}_{3} \mathrm{H}_{2}$ & 1.82 & $\sim 10.70$ & $>0.71$ & .. & .. & .. \\
\hline & $\mathrm{DCN}$ & 2.11 & $\sim 10.43$ & $\geq 0.35$ & & $\cdot$. & .. \\
\hline \multirow[t]{3}{*}{ L1152 } & $\mathrm{CH}_{3} \mathrm{OH}$ & 0.70 & $\sim 9.29$ & $\geq 1.25$ & 0.25 & $\sim 9.29$ & $\geq 0.10$ \\
\hline & $\mathrm{C}_{3} \mathrm{H}_{2}$ & 0.97 & $\sim 10.70$ & $\geq 0.11$ & 0.48 & $\sim 10.70$ & $\geq 0.28$ \\
\hline & $\mathrm{DCN}$ & 0.36 & $\sim 10.43$ & $\geq 0.03$ & .. & .. & .. \\
\hline \multirow[t]{3}{*}{$\mathrm{L} 1152(15,30)$} & $\mathrm{CH}_{3} \mathrm{OH}$ & 0.41 & $16.52(5.21)$ & $1.31(0.44)$ & & .. & .. \\
\hline & $\mathrm{C}_{3} \mathrm{H}_{2}$ & 0.33 & $\sim 10.70$ & $\geq 0.15$ & 0.20 & $\sim 10.70$ & $\geq 0.04$ \\
\hline & $\mathrm{DCN}$ & 0.79 & $\sim 10.43$ & $\geq 0.04$ & .. & .. & .. \\
\hline \multirow[t]{2}{*}{ L1157 } & $\mathrm{CH}_{3} \mathrm{OH}$ & 6.75 & $11.54(1.12)$ & $31.68(4.84)$ & 1.25 & $13.69(3.53)$ & $3.47(1.42)$ \\
\hline & $\mathrm{DCN}$ & 2.56 & $\sim 10.43$ & $\geq 0.16$ & & & \\
\hline $\operatorname{L} 1157(20,-60)$ & $\mathrm{CH}_{3} \mathrm{OH}$ & 6.20 & $8.83(0.45)$ & $64.50(7.99)$ & 3.67 & $8.43(0.88)$ & $28.07(7.37)$ \\
\hline \multirow[t]{3}{*}{ L1174 } & $\mathrm{CH}_{3} \mathrm{OH}$ & 1.01 & $\sim 9.29$ & $\geq 1.47$ & .. & .. & .. \\
\hline & $\mathrm{C}_{3} \mathrm{H}_{2}$ & 0.45 & $\sim 10.70$ & $\geq 0.43$ & .. & .. & .. \\
\hline & $\mathrm{DCN}$ & 1.05 & $\sim 10.43$ & $\geq 0.09$ &.. & .. & .. \\
\hline \multirow[t]{3}{*}{ L1251B } & $\mathrm{CH}_{3} \mathrm{OH}$ & 1.23 & $9.50(0.94)$ & $6.27 \overline{(1.23)}$ & 0.46 & $\sim 9.29$ & $\geq 0.52$ \\
\hline & $\mathrm{C}_{3} \mathrm{H}_{2}$ & 1.01 & $\sim 10.70$ & $\geq 0.60$ & .. & .. & .. \\
\hline & $\mathrm{DCN}$ & 0.84 & $\sim 10.43$ & $\geq 0.09$ & .. & .. & .. \\
\hline $\mathrm{L} 1251 \mathrm{~B}(128,-50)$ & $\mathrm{CH}_{3} \mathrm{OH}$ & 1.78 & $12.15(1.52)$ & $4.50(0.87)$ & .. & .. & .. \\
\hline \multirow[t]{2}{*}{$\mathrm{L} 1251 \mathrm{~B}(-81,85)$} & $\mathrm{CH}_{3} \mathrm{OH}$ & 1.43 & $\sim 9.29$ & $\geq 1.36$ & .. & .. & .. \\
\hline & $\mathrm{C}_{3} \mathrm{H}_{2}$ & 0.90 & $\sim 10.70$ & $\geq 0.17$ & .. & .. & .. \\
\hline \multirow[t]{3}{*}{ CEPE } & $\mathrm{CH}_{3} \mathrm{OH}$ & 6.22 & $13.71(2.90)$ & $10.66 \overline{(2.89)}$ & 2.43 & $16.54(3.38)$ & $5.10(1.27)$ \\
\hline & $\mathrm{C}_{3} \mathrm{H}_{2}$ & 1.01 & $\sim 10.70$ & $\geq 0.07$ & .. & .. & .. \\
\hline & $\mathrm{DCN}$ & 0.87 & $\sim 10.43$ & $\geq 0.07$ & & .. & .. \\
\hline \multirow[t]{2}{*}{$\operatorname{CEPE}(4,17)$} & $\mathrm{CH}_{3} \mathrm{OH}$ & 5.77 & $15.86(2.79)$ & $9.99 \overline{(1.92)}$ & 1.13 & $\sim 9.29$ & $\geq 2.09$ \\
\hline & $\mathrm{C}_{3} \mathrm{H}_{2}$ & 0.78 & $\sim 10.70$ & $\geq 0.13$ & & & \\
\hline \multirow[t]{3}{*}{$\operatorname{CEPE}(-24,-22)$} & $\mathrm{CH}_{3} \mathrm{OH}$ & 9.24 & $10.46(1.41)$ & $6.58 \overline{(1.56)}$ & 0.92 & $\sim 9.29$ & $\geq 0.84$ \\
\hline & $\mathrm{C}_{3} \mathrm{H}_{2}$ & 0.42 & $\sim 10.70$ & $\geq 0.04$ & .. & .. & .. \\
\hline & $\mathrm{DCN}$ & 1.38 & $\sim 10.43$ & $\geq 0.12$ & & .. & .. \\
\hline \multirow[t]{3}{*}{ L1262 } & $\mathrm{CH}_{3} \mathrm{OH}$ & 1.01 & $\sim 9.29$ & $\geq 1.88$ & 0.32 & $\sim 9.29$ & $\geq 1.15$ \\
\hline & $\mathrm{C}_{3} \mathrm{H}_{2}$ & 0.65 & $\sim 10.70$ & $\geq 0.28$ & 0.21 & $\sim 10.70$ & $\geq 0.04$ \\
\hline & DCN & 0.47 & $\sim 10.43$ & $\geq 0.03$ & .. & .. &.- \\
\hline
\end{tabular}

\section{6. $\mathrm{CH}_{3} \mathrm{OH}$ abundances}

From observations of the sources in $\mathrm{C}^{18} \mathrm{O}$, we can calculate the $\mathrm{C}^{18} \mathrm{O}$ column densities, and use these calculations to estimate the $\mathrm{H}_{2}$ column densities, and hence determine $\mathrm{CH}_{3} \mathrm{OH}$ abundances. Assuming optically thin emission, and conditions of LTE, the column density of $\mathrm{C}^{18} \mathrm{O}$ can be calculated using (Bachiller et al. 1990):

$N\left(\mathrm{C}^{18} \mathrm{O}\right)=1.06 \times 10^{13} \times T_{10} \exp \frac{16.5}{T_{10}} \int T_{R}^{*}(1 \rightarrow 0) \mathrm{d} v \cdot(6)$

The $\mathrm{H}_{2}$ column density, in $\mathrm{cm}^{-2}$, can then be estimated (Frerking et al. 1982):

$N\left(H_{2}\right)=5.53 \times 10^{6} N\left(\mathrm{C}^{18} \mathrm{O}\right)+1.22 \times 10^{21}$.

These calculations lead to $\mathrm{C}^{18} \mathrm{O}$ column densities of a few $\times 10^{14} \mathrm{~cm}^{-2}$ to a few $\times 10^{15} \mathrm{~cm}^{-2}$ (Buckle 2001),
$\mathrm{H}_{2}$ column densities of a few $\times 10^{21} \mathrm{~cm}^{-2}$ to a few $\times 10^{22} \mathrm{~cm}^{-2}$, and $\mathrm{CH}_{3} \mathrm{OH}$ abundances, listed in Table 6 , of a few $\times 10^{-10}$ to a few $\times 10^{-8}$ towards the core positions. Where sources have two line components, the broad component typically has a factor of two higher $\mathrm{CH}_{3} \mathrm{OH}$ abundance than the narrow (Fig. 9c, Table 6). A few sources show much larger increases in the $\mathrm{CH}_{3} \mathrm{OH}$ abundances traced by the broad material towards the core positions.

Comparing the linewidths in Table 6, the $\mathrm{CH}_{3} \mathrm{OH}$ linewidths are consistently broader than the $\mathrm{C}^{18} \mathrm{O}$ linewidths, so that the $\mathrm{C}^{18} \mathrm{O}$ is not tracing the high velocity material in the core. This may mean that we are not calculating accurate $\mathrm{CH}_{3} \mathrm{OH}$ fractional abundances in the vicinity of the $\mathrm{CH}_{3} \mathrm{OH}$ emitting regions which give rise to broad linewidths towards the cores.

In low mass star-forming regions, optical depths and column densities are lower for the higher velocity emission 
Table 6. $\mathrm{CH}_{3} \mathrm{OH}$ abundances at the core positions.

\begin{tabular}{|c|c|c|c|c|c|c|c|}
\hline \multirow[t]{2}{*}{ Source } & \multicolumn{3}{|c|}{ Narrow component $\Delta v$} & \multicolumn{3}{|c|}{ Broad component $\Delta v$} & \multirow[b]{2}{*}{$\frac{X(\text { broad })}{X(\text { narrow })}$} \\
\hline & $\begin{array}{r}\mathrm{CH}_{3} \mathrm{OH} \\
\mathrm{km} \mathrm{s}^{-1}\end{array}$ & $\begin{array}{r}\mathrm{C}^{18} \mathrm{O} \\
\mathrm{km} \mathrm{s}^{-1}\end{array}$ & $\begin{array}{r}X \mathrm{CH}_{3} \mathrm{OH} \\
\times 10^{-9}\end{array}$ & $\begin{array}{r}\mathrm{CH}_{3} \mathrm{OH} \\
\mathrm{km} \mathrm{s}^{-1}\end{array}$ & $\begin{array}{r}\mathrm{C}^{18} \mathrm{O} \\
\mathrm{km} \mathrm{s}^{-1}\end{array}$ & $\begin{array}{r}X \mathrm{CH}_{3} \mathrm{OH} \\
\times 10^{-9}\end{array}$ & \\
\hline IRAS 03282 & $1.00(0.05)$ & $0.51(0.19)$ & 7.14 & : & $0.95(0.03)$ & $:$ & : \\
\hline IRAM 04191 & $0.63(0.02)$ & $0.33(0.07)$ & 5.91 & : & $0.72(0.02)$ & $:$ & : \\
\hline L1551 IRS5 & $1.06(0.02)$ & $0.61(0.03)$ & 8.29 & : & $1.07(0.11)$ & : & : \\
\hline L1535 & $0.61(0.07)$ & $0.72(0.02)$ & 2.14 & : & & : & : \\
\hline TMR-1 & $0.55(0.10)$ & $0.40(0.02)$ & 0.84 & : & $0.58(0.01)$ & : & : \\
\hline L1527 & $0.42(0.02)$ & $0.51(0.01)$ & 4.60 & : & : & : & : \\
\hline HH 25 & $0.99(0.05)$ & $0.64(0.03)$ & 9.19 & $3.58(0.20)$ & : & $:$ & : \\
\hline HH111 & $0.72(0.04)$ & $0.83(0.01)$ & 3.62 & : & : & $:$ & : \\
\hline VLA1623 & $1.65(0.07)$ & $0.72(0.04)$ & 4.68 & : & $0.95(0.01)$ & $:$ & : \\
\hline IRAS 16244 & $1.23(0.12)$ & $1.63(0.02)$ & 1.65 & : & & : & : \\
\hline IRAS 16293 & $0.97(0.04)$ & $0.54(0.04)$ & 7.85 & $4.49(0.19)$ & $1.48(0.07)$ & 13.45 & 1.7 \\
\hline S68N & $1.63(0.03)$ & $0.63(0.07)$ & 16.05 & $6.70(0.08)$ & $1.25(0.01)$ & 28.63 & 1.8 \\
\hline L1152 & $0.25(0.13)$ & $0.33(0.09)$ & 0.15 & $0.70(0.08)$ & $0.77(0.05)$ & 1.27 & 8.5 \\
\hline L1157 & $1.25(0.25)$ & $0.37(0.14)$ & 4.89 & $6.75(0.28)$ & $0.45(0.04)$ & 32.35 & 6.6 \\
\hline L1251B & $0.46(0.15)$ & $0.34(0.07)$ & 0.62 & $1.23(0.09$ & $0.70(0.05)$ & 6.33 & 10.2 \\
\hline CepE & $2.43(0.22)$ & $0.44(0.11)$ & 7.42 & $6.22(0.36)$ & $0.74(0.06)$ & 10.81 & 1.5 \\
\hline L1262 & $0.32(0.03)$ & $0.22(0.05)$ & 2.13 & $1.01(0.11)$ & $0.58(0.02)$ & 2.94 & 1.4 \\
\hline
\end{tabular}
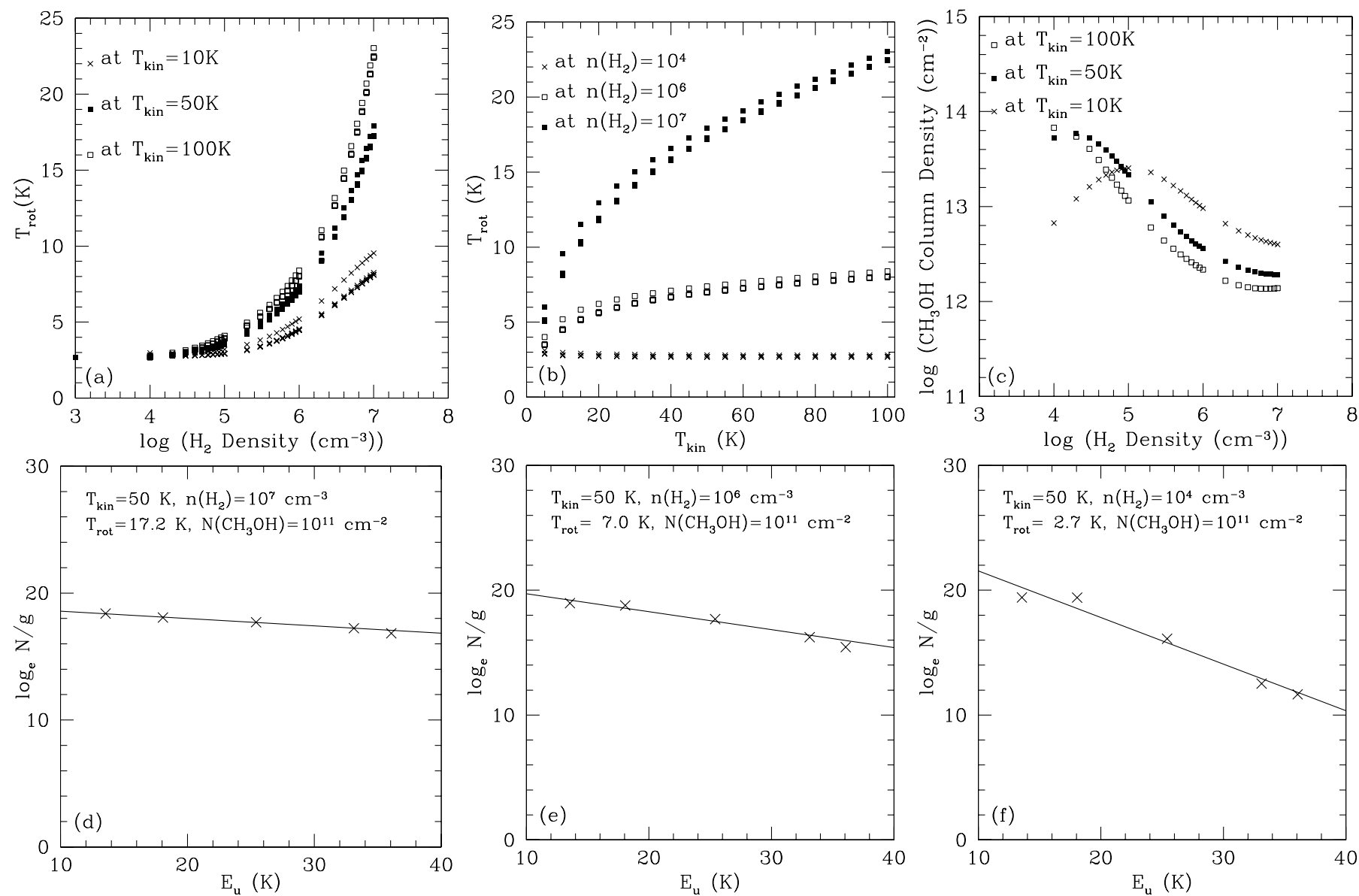

Fig. 8. Results of statistical equilibrium model. a) Variation of rotation temperature $\left(T_{\text {rot }}\right)$ with $\mathrm{H}_{2}$ volume density for kinetic temperatures $\left(T_{\text {kin }}\right)$ of 10,50 and $100 \mathrm{~K}$, and $\mathrm{CH}_{3} \mathrm{OH}$ column densities of $10^{11}-10^{15} \mathrm{~cm}^{-2} . \mathbf{b}$ ) Variation of rotation temperature with kinetic temperature for $\mathrm{H}_{2}$ volume densities of $10^{4}, 10^{6}$ and $10^{7} \mathrm{~cm}^{-3}$, and $\mathrm{CH}_{3} \mathrm{OH}$ column densities of $10^{11}-10^{15} \mathrm{~cm}^{-2}$. c) Variation of column densities, calculated using the rotation diagram technique, with $\mathrm{H}_{2}$ volume density, for kinetic temperatures of 10,50 and $100 \mathrm{~K}$, with a $\mathrm{CH}_{3} \mathrm{OH}$ column density of $10^{13} \mathrm{~cm}^{-2}$. d)-f) Rotation diagrams generated from the model data, for model parameters listed in the plot. As for the observed $\mathrm{CH}_{3} \mathrm{OH}$ lines, all transitions except for the blended $3_{2} \rightarrow 2_{2} E^{+/-}$ transitions have been included in the least squares fit. 
Table 7. $\mathrm{CH}_{3} \mathrm{OH}$ abundances at the outflow positions.

\begin{tabular}{|c|c|c|c|c|c|}
\hline Source & $\begin{array}{r}N\left(\mathrm{H}_{2}\right) \\
\times 10^{20} \mathrm{~cm}^{-2}\end{array}$ & $\begin{array}{r}\Delta v \mathrm{CH}_{3} \mathrm{OH} \\
\mathrm{kms}^{-1}\end{array}$ & $\begin{array}{l}N\left(\mathrm{CH}_{3} \mathrm{OH}\right) \\
\times 10^{13} \mathrm{~cm}^{-2}\end{array}$ & $\begin{array}{r}X\left(\mathrm{CH}_{3} \mathrm{OH}\right) \\
\times 10^{-7}\end{array}$ & $\frac{X(\text { outflow })}{X \text { (core) }}$ \\
\hline HH25 $(224,30)$ & $2.1^{a}$ & 4.58 & 3.7 & 1.8 & 19.6 \\
\hline \multirow[t]{2}{*}{ L1157 (20, -60) } & $4.0^{b}$ & 3.67 & 28.1 & 7.0 & 142.9 \\
\hline & & 6.20 & 64.5 & 16.1 & 49.7 \\
\hline \multirow[t]{2}{*}{ VLA1623 $(45,-24)$} & $0.82^{c}$ & 0.84 & 0.9 & 2.3 & 49.1 \\
\hline & & 1.41 & 5.0 & 6.1 & 130.3 \\
\hline \multirow[t]{2}{*}{ VLA1623 $(-42,16)$} & $0.77^{c}$ & 0.79 & 2.2 & 2.9 & 62.0 \\
\hline & & 1.86 & 11.0 & 14.3 & 305.6 \\
\hline
\end{tabular}

${ }^{a}$ Derived from high velocity CO; Gibb \& Davis (1998).

${ }^{b}$ Derived from CO in this lobe; Umemoto et al. (1992).

${ }^{c}$ Derived from high velocity CO; André et al. (1990).

(Moriarty-Schieven \& Snell 1988; Bachiller et al. 1990; Mitchell et al. 1992). This leads to typical $\mathrm{H}_{2}$ column densities in the high velocity lobes that are at least 1 2 orders of magnitude less than those of the ambient cloud (Bachiller et al. 1990). The lower $\mathrm{H}_{2}$ column densities towards the outflow positions, which are centred on peaks of $\mathrm{CO}$ emission, result in higher $\mathrm{CH}_{3} \mathrm{OH}$ abundances. For example, from our $\mathrm{C}^{18} \mathrm{O}$ observations, we derive $N\left(\mathrm{H}_{2}\right) \sim 10^{22} \mathrm{~cm}^{-2}$ towards the core position of VLA1623, whereas using published $\mathrm{CO}$ data towards the CO outflow we derive $N\left(\mathrm{H}_{2}\right) \sim 10^{20} \mathrm{~cm}^{-2}$ (André et al. 1990). The lower $\mathrm{H}_{2}$ column density at the outflow position leads to abundances at the outflow position that are factors of a few $\times 10-100$ higher than the abundance at the core position (Table 7 ).

For a few sources we have obtained column densities of $\mathrm{H}_{2}$ at our observed outflow position from published high velocity CO data (Gibb \& Davis 1998; Umemoto et al. 1992; André et al. 1990), leading to lower values for the $\mathrm{H}_{2}$ column densities. Table 7 lists the sources, $\mathrm{H}_{2}$ and $\mathrm{CH}_{3} \mathrm{OH}$ column densities, and $\mathrm{CH}_{3} \mathrm{OH}$ abundances for this small sample. The last column gives the ratio of the broad or narrow component $\mathrm{CH}_{3} \mathrm{OH}$ abundance towards the outflow position to that of the same component towards the core position. The smaller values of $\mathrm{H}_{2}$ column densities at the outflow positions leads to higher $\mathrm{CH}_{3} \mathrm{OH}$ abundances at the outflow position by 1 to 2 orders of magnitude over the $\mathrm{CH}_{3} \mathrm{OH}$ abundance at the core position. The abundance of $\mathrm{CH}_{3} \mathrm{OH}$ traced by the broad component material increases by factors of up to 300 towards the outflow positions, while the abundance traced by the narrow component material also increases, by factors of up to 150 towards the outflow positions. The enhanced abundances in material characterised by relatively narrow linewidths implies that the processes involved in increasing the $\mathrm{CH}_{3} \mathrm{OH}$ abundances in star forming regions (discussed in Sect. 5) are as important in the more quiescent envelope regions as they are in the outflowing regions.

\subsection{Origin of enhanced abundances}

The major route to enhance gas phase methanol abundances is through the release of methanol trapped in grain mantles (Charnley et al. 1995; Bachiller et al. 1995; Van Dishoeck et al. 1995). This could occur either through radiative heating of the grains or sputtering. Although a grain temperature of $\sim 90 \mathrm{~K}$ is needed to thermally desorb water or methanol rich ice, the release of $\mathrm{CH}_{3} \mathrm{OH}$ could occur at a much lower temperature, $\sim 20 \mathrm{~K}$, if the methanol is liberated as a minor constituent as the $\mathrm{CO}$ mantle on grains is released (van der Tak 2000). Weak shocks are sufficient to desorb the grain ice mantles: grain-grain velocities of $2 \mathrm{~km} \mathrm{~s}^{-1}$ will shatter ice material, but probably not return much material to the gas phase (Jones et al. 1996). In higher velocity shocks, thermal sputtering takes place, but non-thermal sputtering occurs in low velocity shocks, and is driven by the motion of grains through gas swept up by the shock, or by acceleration of grains in the magnetic field (Jones et al. 1996). This could account for increased abundances of $\mathrm{CH}_{3} \mathrm{OH}$ with relatively narrow linewidths, and does not require high temperatures, nor large relative gas-grain velocities.

Once released, $\mathrm{CH}_{3} \mathrm{OH}$ is destroyed in $\sim 10^{4}$ years in reactions with ions such as $\mathrm{H}_{3}^{+}$(van der Tak 2000), leading to the formation of complex molecules such as $\mathrm{CH}_{3} \mathrm{OCH}_{3}$. This may account for the drop in abundance of $\mathrm{CH}_{3} \mathrm{OH}$ around Class I sources (Sect. 5.2) if the temperatures and densities are sufficient to drive the reactions.

\section{Discussion}

These observations include molecules with differing formation routes, and so we are able to probe several regions within the circumstellar environment, in order to assess the interaction between the outflow and the dense core and envelope surrounding the protostar.

$\mathrm{CH}_{3} \mathrm{OH}$ is one of the more complex molecules found to be abundant in the gas phase in dark clouds (Turner 1998b; Friberg et al. 1988), and is also expected to be 

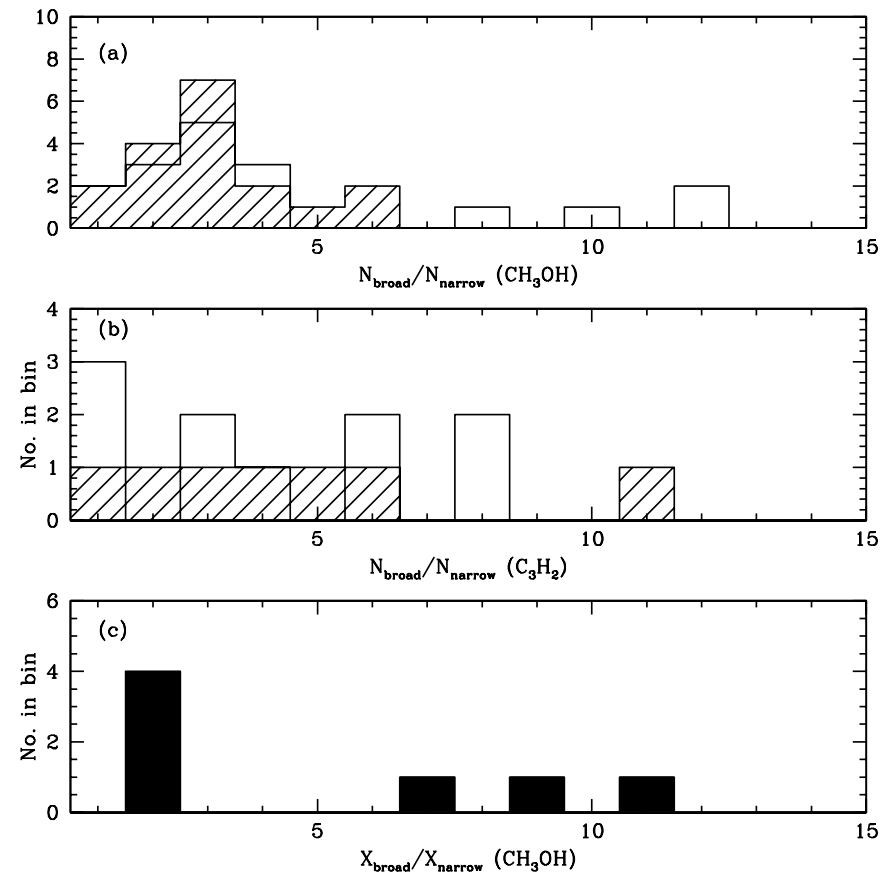

Fig. 9. a) Distribution of $\mathrm{CH}_{3} \mathrm{OH}$ broad to narrow column density ratios. Unshaded histograms show the ratio calculated for core positions, and shaded histograms show the ratio calculated for outflow positions. b) As for a), but for $\mathrm{c}-\mathrm{C}_{3} \mathrm{H}_{2}$ column densities. The broad component material almost always has a higher column density, and core positions generally have larger $\left(\frac{N \text { (broad })}{N \text { (narrow })}\right)$ ratios. c) Distribution of $\mathrm{CH}_{3} \mathrm{OH}$ broad to narrow abundance ratios.

one of the most abundant constituents (Van Dishoeck \& Hogerheijde 1999) of ice mantles in grains in star forming regions. As shocks form from the impact of molecular outflows from protostars with the ambient medium, the gas and dust is heated. The ice mantles evaporate from the grains, and return volatile molecules, including $\mathrm{CH}_{3} \mathrm{OH}$, to the gas phase. Evidence for this can be seen in $\mathrm{BH} 71$, where maps of $\mathrm{CH}_{3} \mathrm{OH}$ and $\mathrm{SiO}$ (a known shock diagnostic) have similar distributions (Garay et al. 1998), suggesting that shocks provide the heating mechanism required to evaporate the mantles.

Transitions of $\mathrm{c}_{-} \mathrm{C}_{3} \mathrm{H}_{2}$ have been detected in emission towards a wide variety of sources (Madden et al. 1989), where the emission usually shows narrow linewidths, with the majority of the emission tracing cold, dense regions: in IRAS 16293-2422, this molecule traces the cold outer part of the envelope (Van Dishoeck et al. 1995). In a few protostellar sources, c- $\mathrm{C}_{3} \mathrm{H}_{2}$ has been observed in outflow lobes (Tafalla \& Myers 1997), with a bipolar distribution, and with shifted, narrow lines without extended wings, suggesting that the $\mathrm{c}_{-} \mathrm{C}_{3} \mathrm{H}_{2}$ is tracing dense clumps of gas that has been dragged into, rather than stirred up by, the outflow.

Although the chemical formation route in quiescent regions is through radiative association reactions, $c-\mathrm{C}_{3} \mathrm{H}_{2}$ can also form in star forming regions through shock chemistry (Lee et al. 1993; Pineau des Forêts et al. 1987;
Neufeld \& Dalgarno 1989). The enhanced c- $\mathrm{C}_{3} \mathrm{H}_{2}$ linewidths observed in W49, a luminous HII region (Matthews \& Irvine 1985), have been associated with emission from an extended, warm region within the acceleration zone of an MHD shock (Pineau des Forêts et al. 1987).

The $\mathrm{c}-\mathrm{C}_{3} \mathrm{H}_{2}$ linewidths derived from our data are similar to those of $\mathrm{NH}_{3}$, a molecule which is abundant in the dense quiescent regions of molecular clouds (Jijina et al. 1999; Benson \& Myers 1989). Jijina et al. (1999) find intrinsic linewidths for $\mathrm{NH}_{3}$ of $0.2-0.6 \mathrm{~km} \mathrm{~s}^{-1}$, towards the low mass star forming regions we have observed, although a few sources in regions of high star-forming activity (such as Serpens) have linewidths up to $0.8 \mathrm{~km} \mathrm{~s}^{-1}$. This is similar to the range of linewidths we find for $\mathrm{c}-\mathrm{C}_{3} \mathrm{H}_{2}$, indicating that this emission, like $\mathrm{NH}_{3}$, is associated with the least disturbed material as previous studies have found (Benson et al. 1994; Lee et al. 1993). The $\mathrm{c}^{-} \mathrm{C}_{3} \mathrm{H}_{2}$ emission is tracing the most quiescent core/envelope gas surrounding the protostar. However, only towards a small fraction of the core positions is this gas detected. The absence of this narrow component towards $70 \%$ of Class 0 core positions implies that the ambient material close to the protostar is disturbed during the very earliest stagest of star formation. Where we detect evidence of strongly disturbed gas from broad $\mathrm{CH}_{3} \mathrm{OH}$ lines the emission from c- $\mathrm{C}_{3} \mathrm{H}_{2}$ is weak.

The low temperatures in low-mass star forming cores in the pre-stellar and collapse stages (Van Dishoeck \& Hogerheijde 1999) leads to significant gas phase enhancement of deuterated species. This is due to their lower ground state energy, so that deuterated species are preferentially formed compared to their hydrogenated counterparts. This also leads to enhancement of deuterated species on grain surfaces, as the accretion of higher abundances of gas-phase deuterated species leads to higher abundances on the grain surfaces. Although to date there has been only one detailed study of deuteration in a low mass source (Van Dishoeck et al. 1995), other low mass sources appear to show similar fractionation (Roberts et al. 2001).

All of the molecules which we have detected towards these low mass sources have also been detected towards quiescent dark clouds; we would therefore expect to detect $\mathrm{CH}_{3} \mathrm{OH}, \mathrm{c}-\mathrm{C}_{3} \mathrm{H}_{2}$ and DCN in the quiescent, unprocessed material surrounding the protostar. The differences between emission arising in dark clouds and protostellar environments can be used to place constraints on the chemical and physical changes which have taken place through the onset of the star formation process.

\subsection{Enhanced abundance regions}

Canonical values for $\mathrm{CH}_{3} \mathrm{OH}$ abundances in dark clouds are of the order a few $\times 10^{-10}$ to a few $\times 10^{-9}$ (e.g. Markwick 2000; Friberg et al. 1988; Turner 1998b). The values of $X\left(\mathrm{CH}_{3} \mathrm{OH}\right)$ of a few $\times 10^{-9}$ to a few $\times 10^{-8}$ 
(Table 6) we determine towards the core positions suggest that the methanol abundance is enhanced by at least an order of magnitude in both the broad and narrow component.

Due to the larger linewidths, we associate the broad component material with shock processing as the outflow impacts ambient circumstellar material. In these regions, the elevated temperatures lead to the evaporation of grain mantles, and increased abundances of volatile molecules such as $\mathrm{CH}_{3} \mathrm{OH}$.

The increased $\mathrm{CH}_{3} \mathrm{OH}$ abundances in material traced by emission with narrow and single component linewidths suggests that the quiescent envelope/core gas has been processed. The quiescent material could be processed through the influence of outflow activity, as the turbulence generated by shocks propagates through the quiescent circumstellar material, although the narrow and single component $\mathrm{CH}_{3} \mathrm{OH}$ lines are narrower than those usually associated with outflow activity.

A second possible mechanism for the processing of quiescent material is through infall activity. The linewidths of the single and narrow component $\mathrm{CH}_{3} \mathrm{OH}$ emission are similar to those usually associated with infall close to the source $\left(0.3-1 \mathrm{~km} \mathrm{~s}^{-1}\right.$, Ohashi et al. 1997; Zhou 1992). However, if the processing were due to infall activity, then we would expect linewidths to decrease at positions further from the source. Generally, we detect similar linewidths in the single and narrow component towards core and outflow positions, although the sources IRAS 16293-2422 and CepE have broader (single or narrow) lines towards the core positions than the outflow positions.

Thirdly, the increased $\mathrm{CH}_{3} \mathrm{OH}$ abundances in material with narrower linewidths could be due to the effect of gas phase chemical evolution (Takakuwa et al. 2000). However, gas phase enhancements of $\mathrm{CH}_{3} \mathrm{OH}$ peak at early stages, and the largest enhancements are seen towards starless cores (Takakuwa et al. 2000). Therefore, enhancements due to gas phase chemical evolution are unlikely to be significant towards sources which have outflows, where enhancement routes include grain mantle evaporation.

\subsection{Class 0 and Class I characteristics}

To look for evidence of evolution in the line properties, the sources have been divided into Class 0 and Class I using values of bolometric temperature obtained from the literature. We have used the criteria of Class 0 with $T_{\text {bol }} \leq$ $70 \mathrm{~K}$, and Class I with $70 \mathrm{~K}<T_{\text {bol }} \leq 250 \mathrm{~K}$ (Table 2).

The $\mathrm{CH}_{3} \mathrm{OH}$ emission from the Class I sources is generally well fit by relatively weak, single component Gaussians. Towards the Class I sources, we do not see extended wings on the $\mathrm{CH}_{3} \mathrm{OH}$ lines, nor strong, narrow components at the source velocity, towards either core or outflow positions. If the Class 0 lines are composed of emission from two distinct regions, the turbulent outflow gas, and the quiescent core/envelope gas, then at the
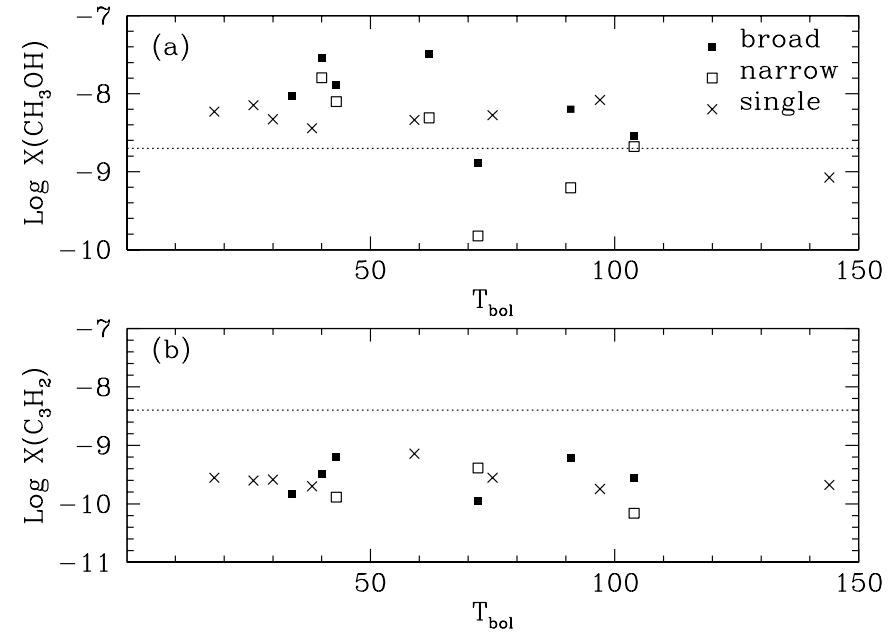

Fig. 10. a) $\mathrm{CH}_{3} \mathrm{OH}$ abundances for the broad, single and narrow components versus bolometric temperature. The horizontal line marks the abundance of $\mathrm{CH}_{3} \mathrm{OH}$ in TMC-1 (Turner et al. 2000; Walmsley 1993; Roberts \& Millar 2000). All of the linewidth components tend to have enhanced abundances with respect to dark cloud values. b) As for a), for c- $\mathrm{C}_{3} \mathrm{H}_{2}$ abundances. For this molecule, none of the linewidth components have enhanced abundances with respect to dark cloud values.

Class I stage, this distinction has all but disappeared: the broad component has disappeared at the Class I stage.

Figure 10 displays the variation in abundance of $\mathrm{CH}_{3} \mathrm{OH}$ and $\mathrm{c}_{-} \mathrm{C}_{3} \mathrm{H}_{2}$ with bolometric temperature. The abundance of $\mathrm{CH}_{3} \mathrm{OH}$ (Fig. 10a) is generally enhanced over dark cloud values for the broad, narrow and single linewidth components. There is weak evidence of a trend of decreasing $\mathrm{CH}_{3} \mathrm{OH}$ abundance with increasing bolometric temperature, with the Class 0 sources having an average value $\left\langle X\left(\mathrm{CH}_{3} \mathrm{OH}\right)\right\rangle=1.5( \pm 0.4) \times 10^{-8}$, whereas the Class I sources have $\left\langle X\left(\mathrm{CH}_{3} \mathrm{OH}\right)\right\rangle=0.4( \pm 0.09) \times 10^{-8}$. These abundance enhancements point to energetic activity, able to affect core material, being at least as, if not more, important at the earliest stages of protostellar evolution as it is at the later stages.

Whether material is disturbed through the action of outflows or possibly infall, the high narrow component $\mathrm{CH}_{3} \mathrm{OH}$ abundances towards Class 0 sources argues that this interaction occurs very rapidly once a central source has formed and that the disturbed gas rapidly dominates the emission. Indeed it appears likely that the bulk of the dense gas around these sources is disturbed as in most of the sources there is little evidence for undisturbed material, the exception being the $\sim 30 \%$ of sources which have narrow c- $\mathrm{C}_{3} \mathrm{H}_{2}$ components.

The apparent decrease in $\mathrm{CH}_{3} \mathrm{OH}$ abundance in the Class I phase implies that the methanol associated with Class 0 sources is removed. One way that a decrease in $\mathrm{CH}_{3} \mathrm{OH}$ abundance could occur is through reactions of $\mathrm{CH}_{3} \mathrm{OH}$ with molecular ions to produce more complex hydrocarbon species (Charnley et al. 1995; van der Tak 2000). Alternatively the methanol rich material may just be removed from the circumstellar region. With a 
velocity of $0.25 \mathrm{kms}^{-1}$ material could leave the beam (in the nearest sources) in $\sim 7 \times 10^{4}$ years and somewhat longer for more distant sources provided it is not gravitationally bound. The decrease in the $\mathrm{CH}_{3} \mathrm{OH}$ abundance also points to a decrease in the rate at which $\mathrm{CH}_{3} \mathrm{OH}$ is released into the gas phase. That the interaction giving rise to the enhancement has lessened by the Class I stage is also shown by the decrease in the narrow component linewidth and the lower incidence of broad components towards Class I core positions. Fuller \& Ladd (2002) have argued on the basis of the evolution of the linewidths towards Class I sources that the outflows in these sources are less well coupled to their circumstellar material than in younger sources. These $\mathrm{CH}_{3} \mathrm{OH}$ results appear to support this interpretation.

Although the $\mathrm{c}-\mathrm{C}_{3} \mathrm{H}_{2}$ abundances are lower limits, the values are at least an order of magnitude below TMC-1 abundances (Fig. 10b) for both Class 0 and Class I sources. The origin of the $\mathrm{c}-\mathrm{C}_{3} \mathrm{H}_{2}$ depletion with respect to quiescent dark cloud values is unclear. $\mathrm{c}-\mathrm{C}_{3} \mathrm{H}_{2}$ abundances can be increased through gas phase reactions in regions with a large carbon abundance (Turner et al. 2000), such as in TMC-1. Alternatively it may be that our derived abundances are underestimated due to the optical depth and excitation temperature differences between the transitions observed here and those typically used for observations of dark clouds. Clearly further modelling of the reaction networks for this molecule in protostellar regions is necessary to understand the evolution of $\mathrm{c}-\mathrm{C}_{3} \mathrm{H}_{2}$ abundances during star formation.

\section{Summary}

We detected $\mathrm{CH}_{3} \mathrm{OH}$ emission towards all of the Class 0 and Class I sources which we observed, with $57 \%$ of the positions observed showing two component line profiles, and with a higher proportion of Class 0 sources showing two component lines than Class I sources. Of the positions observed, $87 \%$ also showed $\mathrm{c}^{-} \mathrm{C}_{3} \mathrm{H}_{2}$ emission with $32 \%$ of these having two component line profiles and $66 \%$ of the sample of sources observed showed DCN emission. Sources which have broader $\mathrm{CH}_{3} \mathrm{OH}$ lines have broader lines in both $\mathrm{c}-\mathrm{C}_{3} \mathrm{H}_{2}$ and $\mathrm{DCN}$. The molecules are all tracing gas moving at similar velocities, as the central velocities of all the emission lines generally differ by only a few tenths of a $\mathrm{km} \mathrm{s}^{-1}$.

A rotation diagram analysis of the $\mathrm{CH}_{3} \mathrm{OH}$ lines gives a rotation temperature $\sim 10 \mathrm{~K}$ toward both core and outflow positions, although statistical equilibrium calculations suggest that the kinetic temperature could be much higher, at $50-100 \mathrm{~K}$. All of the $\mathrm{CH}_{3} \mathrm{OH}$ emission shows enhanced abundances, even the narrower linewidth components. The large abundances of $\mathrm{CH}_{3} \mathrm{OH}$ derived from these data, and the enhancements found towards the outflow positions, suggest that grain mantle evaporation contributes significantly to the chemistry in these regions. The abundance of $\mathrm{CH}_{3} \mathrm{OH}$ in the broad component is enhanced by up to two orders of magnitude, and the narrow and single component has an enhanced abundance of up to an order of magnitude over dark cloud values. The enhanced abundances in material traced by emission with relatively narrow linewidths suggests that much of the circumstellar material has been processed. We detect the broadest $\mathrm{CH}_{3} \mathrm{OH}$ lines towards the core positions rather than the outflow regions, which provides evidence of interaction between the outflow and core material very close to the star.

The narrow linewidth component of $\mathrm{c}_{-} \mathrm{C}_{3} \mathrm{H}_{2}$ has the narrowest linewidth, which is comparable to the intrinsic linewidths detected in $\mathrm{NH}_{3}$, a molecule used to trace quiescent cloud material and so we associate this c- $\mathrm{C}_{3} \mathrm{H}_{2}$ emission with ambient material in the envelope. This component is detected towards both core and outflow positions, in an equal but small fraction of sources, $\sim 30 \%$. We conclude that only a small amount of the circumstellar envelope remains undisturbed, and only a small number of sources contain undisturbed material, at least detectable in this line, even in Class 0 sources.

The broad linewidth component of $\mathrm{CH}_{3} \mathrm{OH}$ we associate with the turbulent regions around the high velocity outflows. Shock heating in these regions leads to evaporation of grain mantles, and large increases in abundances of $\mathrm{CH}_{3} \mathrm{OH}$. The regions influenced by these interactions include positions close to the source, as well as along the outflow axis. The narrow and single emission lines of $\mathrm{CH}_{3} \mathrm{OH}$, the single and broad emission lines of $\mathrm{c}_{-} \mathrm{C}_{3} \mathrm{H}_{2}$, and the emission lines of DCN, appear to be tracing the influence of outflow, or possibly infall activity, on the circumstellar envelope. Increases in $\mathrm{CH}_{3} \mathrm{OH}$ abundances traced by this component indicate that although less energetic, the activity is sufficient to evaporate the grain mantles.

The enhanced abundances and linewidths seen towards the majority of the sources show that the energetic activities that begin during star formation, particularly outflow but possibly also infall related, very quickly affect the circumstellar envelope surrounding a source. These interactions dominate the emission from Class 0 sources, even for lines which are expected to trace the quiescent material, such as $\mathrm{c}-\mathrm{C}_{3} \mathrm{H}_{2}$. Evidence from the decreased abundances and the rarity of two component lines towards the core positions in Class I sources point to less activity in these sources than in the Class 0 phase. This suggests that the interaction between the outflow and the circumstellar material has significantly decreased by the time sources reach the Class I phase.

Acknowledgements. Astronomy at UMIST is supported by PPARC. J.V.B. would like to acknowledge the financial support of a PPARC studentship. The authors would like to thank Prof. T. J. Millar for discussions on the origin of enhanced $\mathrm{CH}_{3} \mathrm{OH}$ abundances, and NRAO staff for their help and advice.

The National Radio Astronomy Observatory is a facility of the National Science Foundation operated under cooperative agreement by Associated Universities, Inc. 


\section{References}

Anderson, T., De Lucia, F. C., \& Herbst, E. 1990, ApJS, 72, 797

André, Ph. 2000, private communication

André, Ph., \& Bacmann, A. 1999, ApJ, 513, L57

André, Ph., Martin-Pintado, J., Despois, D., \& Montmerle, T. 1990, A\&A, 236, 180

Bachiller, R., Codella, C., Colomer, F., et al. 1998, A\&A, 335, 266

Bachiller, R., \& Gutiérrez, M. P. 1997, ApJ, 487, L93

Bachiller, R., Liechti, S., Walmsley, C. M., et al. 1995, A\&A, 295, L51

Bachiller, R., Martin-Pintado, J., Tafalla, M., et al. 1990, A\&A, 231, 174

Benson, P. J., \& Myers, P. C. 1989, ApJS, 71, 89

Benson, P. J., Caselli, P., Myers, P. C., et al. 1994, in $\mathrm{N}_{2} \mathrm{H}^{+}$ and $\mathrm{c}^{-} \mathrm{C}_{3} \mathrm{H}_{2}$ in Dense Cores, ed. D., Clemens, R., Barvainis, Clouds, Cores and Low Mass Stars, ASP, 65, 67

Buckle, J. V. 2001, in Observational Studies of Low Mass Star Formation, Ph.D. Thesis, Physics Dept., UMIST, Manchester, UK

Buckle, J. V., \& Fuller, G. A. 2001, in prep.

Charnley, S. B., Kress, M. E., Tielens, A. G. G. M., et al. 1995, ApJ, 448, 232

Chernin, L. M., \& Masson, C. R. 1995, ApJ, 455, 182

Cox, P., Walmsley, C. M., \& Güsten, R. 1989, A\&A, 209, 382

Friberg, P., Madden, S. C., Hjalmarson, A., \& Irvine, W. M. 1988, A\&A, 195,281

Frerking, M. A., Langer, W. D., \& Wilson, R. W. 1982, ApJ, 262,590

Fuller, G. A., \& Ladd, E. F. 2002, ApJ, in press

Garay, G., Köhnenkamp, I., Bourke, T. L., et al. 1998, ApJ, 509, 768

Gibb, A. G., \& Davis, C. J. 1998, MNRAS, 298, 644

Haque, S. S., Lees, R. M., Saint Clair, J. M., \& Beers, Y. 1974, ApJ, 187, L15

Hatchell, J., Fuller, G. A., \& Ladd, E. F. 1999, A\&A, 344, 687

Hatchell, J., Thompson, M. A., Millar, T. J., \& Macdonald, G. H. 1998, A\&A, 338, 713

Hayashi, M., Hasegawa, T., Ohashi, N., \& Sunada, K. 1994, ApJ, 426, 234

Helmich, F. P., Jansen, D. J., de Graauw, Th., et al. 1994, A\&A, 283, 626

Jijina, J., Myers, P. C., \& Adams, F. C. 1999, ApJS, 125, 161

Johnston, K. J., Gaume, R., Stolovy, S., et al. 1992, ApJ, 385, 232

Jones, A. P., Tielens, A. G. G. M., \& Hollenbach, D. J. 1996, ApJ, 469, 740

Kalenskii, S. V., Dzura, A. M., Booth, R. S., et al. 1997, A\&A, 321,311

Lada, C. J. 1982, High Velocity Flows and Molecular Jets, in Submillimetre Wave Astronomy(Cambridge University Press) 175

Lada, C. J. 1985, ARA\&A, 23, 267

Lee, C. W., Minh, Y. C., \& Irvine, W. M. 1993, JKAS, 26, 73

Madden, S. C., Irvine, W. M., Matthews, H. E., et al. 1989, AJ, 97, 1403

Mardones, D., Myers, P. C., Tafalla, M., et al. 1997, ApJ, 489, 719
Markwick, A. J. 2000, in Chemistry in Dynamically Evolving Astrophysical Regions, Ph.D. Thesis, Physics Dept., UMIST

Masson, C. R., \& Chernin, L. M. 1993, ApJ, 414, 230

Matthews, H. E., \& Irvine, W. M. 1985, ApJ, 298, 61

Menten, K. M., Walmsley, C. M., Henkel, C., \& Wilson, T. L. 1986, A\&A, 157, 318

Mitchell, G. F., Tasegawa, T. I., \& Schella, J. 1992, ApJ, 386, 604

Moriarty-Schieven, G. H., \& Snell, R. L. 1988, ApJ, 332, 364

Myers, P. C., Bachiller, R., Caselli, P., et al. 1995, ApJ, 449, L65

Neufeld, D. A., \& Dalgarno, A. 1989, ApJ, 340, 869

Ohashi, N., Hayashi, M., Ho, P. T. P., \& Momose, M. 1997, ApJ, 475, 211

Parker, N. D., Padman, R., \& Scott, P. F. 1991, MNRAS, 252, 442

Pickett, H. M., Poynter, R. L., Cohen, E. A., et al. 1998, in Submillimeter, Millimeter, and Microwave Spectral Line Catalog, J. Quant. Spectrosc., Rad. Transfer 60, 883

Pineau des Forêts, G., Flower, D. R., Hartquist, T. W., \& Millar, T. J. 1987, MNRAS, 227, 993

Richer, J., Shepherd, D., Cabrit, S., et al. 2000, in Molecular Outflows from Young Stellar Objects, ed. V., Mannings, A. P., Boss, S. S., \& Russell. Protostars and Planets IV (Unversity of Arizona Press), 867

Roberts, H. R., Fuller, G. A., Hatchell, J., et al. 2001, in prep. Roberts, H. R., \& Millar, T. J. 2000, A\&A, 361, 388

Stahler, S. W. 1994, ApJ, 422, 616

Shu, F. H., Najita, J., Ostriker, E., et al. 1994, ApJ, 429, 781

Shu, F. H. 1977, ApJ, 214, 488

Tafalla, M., \& Myers, P. C. 1997, ApJ, 491, 653

Takakuwa, S., Mikami, H., \& Saito, M. 1998, ApJ, 501, 723

Takakuwa, S., Mikami, H., Saito, M., \& Hirano, N. 2000, ApJ, 542,367

Terebey, S., Padgett, D. L. 1996, in Millimetre Interferometry of Class 0 Sources, ed. B., Reipurth, C., Bertout, Proc. IAU Symp. 182, Herbig-Haro Flows and the Birth of Stars (Kluwer Academic Publishers), 507

Terebey, S., Beichman, C. A., Gautier, T. N., \& Hester, J. J. 1990, ApJ, 362, L63

Thompson, M. A., Macdonald, G. H., \& Millar, T. J. 1999, A\&A, 342, 809

Turner, B. E., Herbst, E., \& Terzieva, R. 2000, ApJS, 126, 427

Turner, B. E., Lee, H.-H., \& Herbst, E. 1998, ApJS, 115,91

Turner, B. E. 1998, ApJ, 501, 748

Umemoto, T., Iwata, T., Fukui, Y., et al. 1992, ApJ, 392, L83

van der Tak, F. 2000, in The Embedded Phase of Massive Star Formation, Ph.D. Thesis, University of Leiden

Van Dishoeck, E. F., \& Hogerheijde, M. 1999, Models and Observations of the Chemistry Near Young Stellar Objects, in ed. C.J., Lada, N.D., \& Kylafis, The Origin of Stars and Planetary Systems (Kluwer Academic Publishers), 97

Van Dishoeck, E. F., Blake, G. A., Jansen, D. J., et al. 1995, ApJ, 447, 760

Walker, C. K., Narayanan, G., \& Boss, A. P. 1994, ApJ, 431, 767

Walmsley, C. M. 1993, J. Chem. Soc. Faraday Trans. 89, 2119

Zhou, S. 1992, ApJ, 394, 204 